\title{
ZUR
}

\section{GESCHICHTE DER VERBINDUNGEN EINES $S$ BEZ. SCH MIT EINEM CONSONANTEN IM NEUHOCHDEUTSCHEN.}

I.

$\S$ 1. Die alten consonantenverbindungen $s l, s m, s n, s p, s t$, $s n$ werden wortanlautend im nhd. als $s l, \check{s} m$, šn, šp, št, šn ge. sprochen und auch die schrift suchte dieser aussprache wenigstens bei $\operatorname{schl}, s c h m, s c h n, s c h w$ gerecht zu werden. Ueber die zeitliche und örtliche ausdehnung dieser schreibung soll die folgende zusammenstellung einen uberblick gewähren.

Als zeitliche grenze nach rlickwärts ist, wie aus den grammatiken (Weinhold, Mhd. gr. ${ }^{2} \S 206$. AG. $\S 190$ ) erhellt, das ausgehende 13. jabrh., als zeitliche grenze nach vorne, wie es aus den bestimmungen bei Kolross, 1) Fab. Frangk, ${ }^{2}$ )

1) In seinem Enchiridion (Müller $80 \mathrm{ff}$.) sagt er: 'By di/ser kürlzung solt du ouch wissen / das offt der büchstab s. allein für sch. geschriben würt / vnd dasselbig geschicht gemeingklich in den worten! in welchen nach dem sch. difse biichstaben/ namlich l. m. n. $r$. und $w$ von stund. an volgend / wie du in nachgesetzter figur söllichs eigentlich vnd heyter sähen würst.'

$$
\text { s für sch }\left\{\begin{array}{l}
l \\
m \\
n \\
r \\
w
\end{array}\right\} \text { slahen (slemmen) }
$$

2) In seiner Orthographia (Miiller 104) heisst es: Widderumb aber ist dis kein abbruch / sondern für gnug / vnd ein zierd angenohmen / so weilands ettliche buchstaben vbersehen werden / als das ch jnn diesen vnnd der gleichen / sprach / sprech / storch / straus / für schprach / schprecht / 
und Jo. Hel. Meichssner ${ }^{1}$ ) bervorgeht, das beginnende 16. jh. anzusetzen. Durch den begriff 'hochdeutsch' ist auch die örtliche begrenzung erschöpft.

Von vornherein mahnt die orthographische manier der nhd. schriftsprache die erwähnten verbindungen in zwei gruppen zu scheiden, einmal $s l, s m, s n, s w$, die als $s c h l, s c h m, s c h n$, $s c h w$ geschrieben werden, zum andernmal $s p$, st, bei denen an der schreibung zäh festgehalten wird. Hiezu kommt noch, dass die verbindungen der ersten gruppe im gegensatz zur zweiten im inlaute fast gar nicht erscheinen.

\section{A. sl, sm, sn, sw.}

\section{I. sl.}

1) In Oberdeutschland:

a) In Alemannien.

1. Urkundliche belege:

§2. G Iarus. Hofslang $1289 ;$ keiner schlachte 1302; beslossen (3) 1340; tot schlag (2), totslag 1350; ab schlag 1359; erslagen 1386; beslossen, slachen 1393; dry schlagkne 1412 no. 144; todsleg 1419 no. 160; fürslahen, abze schlahen 1419 no. 170 ; n. pr.: Schlipföwer, Schlipffouwer Schlüsse neben Slüsse 1421 no. 167; ussgeslossen 1424; slachen (ö), geslagen (i) 1425 no. 175 ; sloss, besliessen 1425 no. 176 ; verschlicht, beschlossen 1428; todschlag 1429 n. 185: geschlagen, abschlag 1429 no. 186 ; schlössern (ö) 1437 no. 201; sloss (ï), beslossen, besliessung, todsleg, verricht und verslicht 1437 no. 202. 205; schloss (2), slossern, schlossen, slossen (ö), abschlahen 1437 no. 206; schlossen, schloss, ufschlag 1437 no. 207; schloss (8) 1437 no. 209; sloss (2) 1437 no. 212; sloss (2) 1438 no. 216; schlichten, beschliessung, beschliessen, abschlachen, verschlicht 1438 no. 218; beschlossen, ent schlachent (2),

$s(c h) t(h) r a u / s$. (Hier hat die Frankfurter ausgabe schtrau/s, die Wittembergische und die Kölnische sthrau/s. Ueber dieses sth s. unten $\$ 23$, anm.). Vnd ist E'in gemeine Regel. Wenns $p$ odder $t$ nach dem sch / von rechte gefordert / so wirds ch vermieden / vnd das $p$ odder $t$ schlechts zunehst ans s gesatzt / wie jtzt gesehen. Des gleichen haldens ettlich auch $i$ mit dem $l$ und $w$ wenn sie nach dem sch gehen / das sie das ch meiden / vnd schreiben also Slesier / Sweidnilz | Sweitzer / für Schlesier | Schweidnitz / Schweitzer etc. / Welches aber nicht so passt als jens / jnn vbung ist.

1) In dessen 'Handbüchlein' heisst es (Müller s. 163): Es mag ouch vilmaln ch vermitten vnd geschriben werden / Swalbach / Swytzer / slag | slafftrunck / vnnd nit Schwalbach / Schwytzer etc.

$\left.{ }^{2}\right)$ Ueber die seltenen $s l, s m, s n, s w$ im inlaute s. unten $\S 32$. 
ent schlagen 1439 no. 221 ; ussgeschlossen 1440 no. 224; slossen (2) 1440 no. 225; beschlossen, beschliessen, verschlichti sach, todschleg, verslagen oder verspert 1440 no. 233. - Ba s el: ${ }^{1}$ ) von Sliengen 1284 no. 157; von Sliengen, zem Slüssel 1332 no. 293; geslagen 1334 no. 297; von Sliengen 1337 no. 303,1342 no. 317 ; von Schliengen 1347 no. 335 ; Slopen 1348 no. 337 lat. urk.; slaht 1350 no. 344; von Sliengen 1356 no. 364; geschlechten 1357 no. 367 ; slahen 1359 no. 377 ; slahen s. 406,3 ; abeslag s. $406,5.1371$ no. 412 ; geslagen 8. 411 , 24. a. 1371 no. 415 ; am sliffe s. 415 , 15. 16 ; am sliphe s. 416,3 . a. 1371 no. 416 ; einheinrslahte s. 425,34 ; $a b$ slahen $426,6.1373$ no. 420 ; abslag s. 437 , 3. a. 1374 no. 428; ent schlagen s. 446. a. 1380 no. 438 ; Heinrich von Schleyten 8. 467, 19; schlechter s. 468 , 4. a. 1384 no. 455 ; dem sleyrun s. 472 , 12. a. 1381 no. 457 ; totslege s. 481, 14 ; abslahende s. 481, 28. a. 1386 no. 468 ; Slüppe 1390 no. 475; an dem schliff s. 527, 26, ze Schlugken s. 528,5, ze Slugken 8. 528,6. 8. a. 1392 no. 486 ; abslag (2) 1393 no. 489; geslagen s. 543, 8. a. 1395 no. 496; Schlegel s. 544, 21, entschliuge s. 544, 38, entschlahen s. 550,12, entschlagen s. 550,13. a. 1396 no. 499 ; ufges chlagen s. 555, 2, ufgeslagen (3), ufzesiahende 8. 555, 17. a. 1397 nu. 502; entslahen s. 571, 6, entslahende s. 571, 7, a. 1399 no. 512; uf dieselben schlosse s. 589, 37, ges chlagen ib. a. 1400 no. 525; slosse, slossen, schlossen 8. 594. 2. 1400 no. 526; uff schlahen s. 621,29 . a. 1406 no. 548; beslossen s. 703, 10. a. 1417 no. 601 ; geslicht 1417 no. 603 ; slahen s. $720,14.2 .1421$ no. 616 ; verslossen, todslag s. 734 a. 1422 no. 627 ; slacht s. 736,15 , todslag s. 737,16 u. ö., erslagen s. 739,3 a. 1422 no. 628 ; beslagen s. 748,29 a. 1422 no. 637 ; erslagene s. 756,18 , todslage s. 753,35 u. ö., tod schlage s. 754,13 a. 1423 no. 640 ; in den Schlatthof s. 768, 27 a. 1424 no. 645; geslicht s. 787, 7 a. 1431 no.661; die slosse 8. 788,14 , ze slahende 8. 791, 4, züslahunge 8. 791, 9, abeslag s. 792, 2 a. 1431 no. 664 ; todslagk s. 806, 36 u. 8., geslagen 8. 807, 2, ufslahen s. 808, 6, beslossen s. 808, 9 a. 1435 no. 677; geslagen s. 812, 27 a. 1436 no. 682 ; Sluzz s. 818, 29 a. 1437 no. 687 ; abeslag s. 820, 28 8. 1438 no. 689 ; sleyffin s. 826,31 a. 1438 no. 692 ; geslagen, verslúge s. 858 a.1445 no. 722. - Weistümer: Eigen (Rochholtz s. 4) nur sl (vor 1313); erschlagen (öffnung von Berkon 1348 ib. s. 21); nur schl im weistum v. Ermatingen (Grimm 1, 239 ca. 1350); slahen (Birmenstorff 1363, Rochholtz s. 44); schlüeg (Bffnung von hudolfstetten nach 1408, ib. s. 59); nur schl im weistum v. Altorf (Grimm 1,11, a. 1439).

\section{Belege aus literarischen denkmälern: ${ }^{2}$ )}

8 3. a) Mainauer naturlehre: Nor sl: slecht s. 1; besluzit 2; slafene 2. - Basler Nibelungenbruchstiicke: Nur schl, vgl. Wackernagel s. 45. - Der maget krône: 5 sl: slafen 2, 46; geslacht 4, 24; slo/s *4, 394; beslafen 5, 68; slahen 5,235 gegen 14 schl: schlofen 3,2 ; schlahen 3,27 ; erschlugen $* 3,128$; schlug 4,211 . 4, 486; geschlagen 4, 246. 4, 474; schleif 4, 291; beschlossen 4, 299; erschlagen $* 4,343$; ge-

1) Bei benutzung der urkunden wurden nur originale beachtet.

2) Die anordnung ist chronologisch (wenigstens ungefähr). 
schlagen 5,239 ; schlos $*_{5}, 247$; schlig 5,314 ; schlies 5,382 . - H u go v o Mon tf ort: schreiber A: 9 sl: slachte ${ }^{*} 1,11 .{ }^{*} 4,34 .{ }^{*}, 351$; ver slossen $*_{1}, 83$; umbeslossen $1,66.5,143$; slechte 3,71 ; slechter 4,44 ; slichen *8, 2 gegen 1 schl: geschlichen 5,71; schreiber B: 4 sl: zerslagen ${ }^{*} 13,5 ;$ beslossen 27,28 . 31,152 ; sleht $* 28,235$ gegen 49 schl: beschlossen 13, 58 . 28,542 . 36, 6; beschlossen 18, 5. 176. 26, 12. 27, 10. 40. 34, 28; geschlossen 28,240 ; beschliessent 21,16 ; beschlüst 35,$20 ;$ geschlaffen 15,$2 ;$ schlaffen 29, 127. 22, 100. 34, 40. 35, 21 ; entschlaff 18,5 ; entschlieff $25,13.47$. 85. 147 ; schlaff 28,708 ; schlacht 15,16 ; schlecht 27,83 ; schlechst 27,71 ; schlahent $26,41 .{ }^{*} 29,19$; schluog ${ }^{*} 15,96.25,27.94$; beschlagen 28,27 ; geschlagen 28,142 ; erschlagen ${ }^{*} 24,24 .{ }^{*} 60 .{ }^{*} 76$; schlach 29,45 ; schliemen 27,114 ; schlicht $28,176.31,28$; schlichte $29,4.32,152$; schlichten $* 31,143$; schlecht ${ }^{*} 29,95.31,44$. 52. 35, 11; schlichen 31,203 ; geschlecht 33,145 ; schreiber C: nur schl: schlichen 18,$24 ;$ schliessest ib. $100 ;$ schlaffen $*_{\mathrm{ib}}$. 104; schlaff ib. 180 . - K u ch imeister: 22 sl: erslagen $*(17)$; zerslugent s. *106; sliggen 193.289 ; zersligg s. *204; slahen 289 gegen 2 schl: beschlagen 139 ; schlitten 331 . - Stretlinger chronik: fast nur sl: slo/s 11, 13,15. 21. 14 ö., 19; geslicht 2, 122 u. o.; slaf 9, 2. 3; entslief 9,3 ; slafen 9,13 ; slafen 28,5 ; slach 8,10 u. 8. w. gegen 1 schl: die schlü/sel s. 105, 20. - Richental: nur schl.

B) Merswin: 1 sl: slecht s. 41, gegen sonstiges schl (19 mal). Elsässische predigten (hg. von Birlinger, Alem. 1, $60 \mathrm{ff} .186 \mathrm{ff}$. $225 \mathrm{ff}$.): sl: fürslinden ${ }^{*} 69$; fürslinde ${ }^{*} 71$ gegen sonst schl: schlossen 187 ; entschlaffen, schlafen ib.; schloses ib.; beschlossen 188; schlaugen, schlaugent 191; schlahent ib.; geschluogen ib.; schluog 192; schlag 228 u. s. w. Das Elsässische arzneibuch, die Erläuterung der regel des hl. Augustin (hs. 2966 der Wiener hofbibliothek, von 1459 ?), das Hl. namenbuch: nur sl.

y) Augsburger stadtbuch: nur sl. - Wackernagels predigten: uberwiegend schl (vgl. Weinhold, Die sprache in W. Wackernagels altdeutschen predigten s.7. 10. 15. - Ingold: 1 sl: sluf s. 80; unsslicht s. 25, 10. - Conr. von Weinsbergs einnahmen- und ausgaben-register: nur schl: schlos s. 16; beschlagen 8.17 . - 0 tt $\mathrm{Rulands}$ manuale: 8 sl: beslachen s. 1 ; umslahen 8. 16 (2); umgeslagen s.17; umbslag s. 21. 26 (2); umbslagen s. 32 gegen 2 schl: beschlachen s. 1. 17; umbschlagen 8. 18. - Hermann von Sachsenheim, Mörin: 27 sl: entslieff v. 63; uff slag 848 ; hersluog ${ }^{*} 1019$; slecht $*_{1196 .}{ }^{*} 1252.1506$. 1542. 2202. 2407. 2530. 3458; besliessen 1254; pslie/s 2072; verslossen *2165; sluog 2702. 1793. 4106. 4961; slachen 2648. 2714. 2817; geslagen 2722. 4637; geslecht 3207 . 4053; slief 3250 ; slos 4034 gegen 56 schl: schlüssel 121; schlü/s 138. 3138; entschlauffen 173; schlo/s 191; 4944. entschlieffen 209; schlach 2i4. 381. 1956. 2404. 4998; schluog ${ }^{*} 501 .{ }^{*} 4500$. 4531. 4851; schlahen 543. 3269. 3244. 4187. 5607; geschlagen 813; beschlossen 1108. 4287; abschlahen 1719; beschliessen 2042; schlust 2056. 4789; schlauffens 2856; ufschlüssen 2946; schlauffle 3027 ; schlauffen 3053. 3243. 3623. 4547. 4743; geschlauffen 3239; beschlos 3765 ; schlichen 3870. 4064. 5637; schlechten 3878; geschliffen 4209; beschliesst 4352 . 
5246 ; schlecht 4349. 4990. 5526 ; überschlahen * 4745 ; schlaff (v.) 4751 ; schlewern 5006; schlieffen 5214; schlauff 5721; schlieff 5854; verschlossen *6053. - Der goldene tempel und Jesus der arzt: nur schl. Ka ufringer: 64 sl: beslagen 1,139. 152. 232. 305; erslagen *2,95. 16, 115 ; slag 2, 254. 11, 278. *16,45; swerlzsleg 4, 172; slachen 3,581 ; slahen $*_{14}, 132$; slach 16,183 ; slecht 14,$748 ;$ sluog $* 3,1100.452 .17,281$; sligen 3, 505. 14,408; slagprugg 14, 193; absleg 16, 432; geslagen 3, 483. 642. 695; underslagen *14, 258; geslichen 2, 127; sleicht 4,178; beslaffen 2, 132. 5, 185 ; beslauffen 15,639 ; beslieff $5,311.450$; slaffen 11,403 ; slauffen 15,$25 ;$ slauffens ${ }^{*} 2,214 ;$ slauffen 1 . pl. ${ }^{*} 8,214 ;$ slieff $3,579.14,594 ;$ slieffen 5, 258; slauff 14, 251. 574; entslauffen 14, 263. 629; entslaffen 14, 585; entslieff 11,304 ; beslos 2,188 . $7,236.8,186.11,216$. 14, 598; beslossen 16,76 ; geslossen 16,98; verslos *4, 285; slos 8,364 ; slüssel 14, 190; slecht 12, 56; geslächt 4, 61. 99. 123. 139. 8, 20. 14,21; geslecht 4,365. 383 gegen 22 schl: schlauffen 1,71. 304 ; schlaffen 8,240 ; schlauffens $* 5,396$; schlauf-

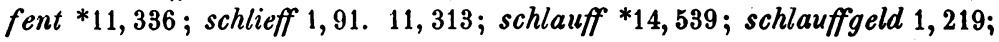
schlos 11,226 . (v.); schlos (8.) 5, 634; schlecht 1, 384. *395. *5, 562. 15, 96 ; schleicht 2,286 ; schleichen 16,602; geschlächt 4, 128. 8, 318.391; entschleift $16,232$.

b) Im bairisch-österreichischem sprachgebiet.

1. Urk undliche belege:

$\$ 4$. Ottenslage 1305, Urkdb. des landes ob der Enns bd. 4, 495 no. 531; Slierbach 1306 ib. s. 499 no. 535; Neit schlag 1312 ib. 5, 74 lat. urk.; Neitslag 1322 io. 5, 346 no. 329, lat. urk.; Otten dez Slegels 1359 ib. 7 s. 625 ; geschlechtes 1361 ib. 8 s. 28 u. 31 , no. 30 ; slechten 1361 ib. 8 no. 42 ; geslecht 1364 ib. s. 194 no. 191 ; slahen, geslagen 1369 ib. s. 419 no. 423; geslossern (castellis) 1370 ib. s. 456 ; inbeslossen 1370 ib. s. 473 ; todsleg 1371 ib. s. 510. - Aus weistümern: todtslag 1342, bergrechte in der Gastein und Rauris Ö. w. 1 8. 200; totschlach ib.; durchsleg ib. s. 196; totslag 1358, Lungau ib. s. 238; totschlag 1462, Tiroler w. 1,53, Frauenchiemsee im gebirg (ö); lotstag (ठ̋) 1462 ib. s. 253, Axams; erslagen, slaffenden, sligg 1487, landrecht im Zillertal Ö. w. 1,317; geslagen (¿), slahen, furslahten, erslagen, slecht, sligg, slachrecht, fürslag, fürschlacht, schlagen (2), geschlouss 1497 und 1498, Mittersill ö. w. $1,283 \mathrm{ff}$.

2. Belege aus literarischendenkmälern:

§ 5. Oswald von Wolkenstein: überwiegend sl: im ganzen 130, wenn man auch die in den niederdeutschen liedern vorkommenden mitrechnet, wie slapp 56, 1. 7 gegen 2 schl: schlegel 5, 5. 13; schlicklin 27, 2. 1. - Peter Suchenwirt: nur sl. - Hans Schiltherger 1 sl: schlo/s s. 111, 21 gegen sonstiges schl. - Nürnberger polizeiordnungen (13. u. 14. jh.) wechsel zwischen sl und schl. Tucher: erste hand 159 sl: beslossen 18, 9. 297, 3; besloss 211, 2. 15; geslossen 267,34 ; verslossen $* 287,29$; slo/s 111,17 . 158, 10. 27. 159, 11. 160, 13. 21. 161, 2. 4. 11. 21. 25. 31. 162, 1. 16. 20. 23. 25. 26. 30. 33. 34. 35. 248, 20. 22. 33. 249,1 . *7; slossen 150,1 . 159, 4. 160, 7. 20. 161, 26. 246, 5. 248, 28 ; mahelslo/s 211, 9. 15. 26. 212, 2. 16. 21. 28. 213, 7. 24. 35. 
214, 6. 32. 215, 2. 266, 9; keltenslo/s 292, 26; slosser 97, 25. 248, 10. *19. *26. *249, 5; slossern 242,20 ; slo/su'ein *40, 28 ; slussel $221,25$. 222 , 10. 223, 11. 224, 31. *248, 19. 25. 25i, 26. 31. 258, 5. 265, 7. 15. 266, 10. 287,33 ; slusselen 248,29 ; Slussclfelderin $* 259,32$; Slusselfelder 264, 17. 31; abgeslagen 38, 3. 200, 31. 231, 33. 244, 7; abslagen 210, 29. 221,13 . 232, 5 ; abslahen 218,8 . 230,5. 6. 232, 34; abzuslahen 218, 18 ; abslecht 216, 24. 239,4; anslecht 147, 21. 26; anslahen 262, 17 ; aufslach 256,33 ; furslahen ${ }^{*} 251,24$; überslahen $*_{236,36}$; verslahen $*_{267}, 26$; slagen 40,14. 45, 33. 94,9. 217,6; slahen 234,15. 256, 9; slachen *201,30; slecht 216,27. 235, 2. 256,13. 275, 23 ; slugen 255, 6; geslagen 64, 17. 94, 3. 5. 230,14 . 287,24 . 297,14 . 289 , 7 ; slach $* 245,30$; slachglocken 246,2 ; slachhaus 199,32. 200, 1. 2. 19; ofterslegen *77,30; durchsleg 288, 36; huntslaher 267, 24. 271,1; messingslaher 95, 23. 152, 7. 153,33. 154, 3. 7. 19. 161, 4. 17; 191, 8; pecksluher 157,6; slegel 217,31). 31. 218, 5. 219, 29. 220,3. 8. 16. 233, 25. 35; slegelziehens 234,2; Slegels 193,10; geslicht 59,25 ; slecht ${ }^{*} 291,21$; slechtz 45 , 7 ; slechten 211,6 ; snur slechtz $*_{166,12}$;

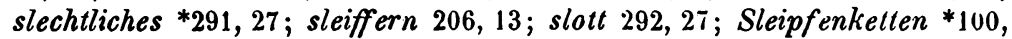
14 ; Slemel *162, 16 gegen 433 schl: geschlossens 95,13 ; schleust 128, 21. 129,14 ; verschlie $\int *_{1} 28,15$; schlo/s 96, 5. 9. 21. 29. 97, 2. 101, 10. 127, 12. 128,26 . $150,2 . * 4$. 5. 6. 7. 9. 10. 11. 13. 15. 16. 18. 20. 15(1, 22. 24. 26. 28. *30. 151, 1. 3. 5. 7. 9. 11. 13. 15. 17. 19. 21. 23. 25. 27. 29. 31. 33. 152, 1. 3. 5. $[$ shlo/s] 7. 9. 10. 12. 14. 16. 18. 20. 22. 24. 26. *28. 30. 31 . 33. 35. 37. 153,1 . 3. 5. 7. 9. 11. 13. 15. 17. 19. 21. 23. 25. $27 . *^{*} 29$. *31. 33. 154,1 . 3. 5. 7. 9. 11. 13. 15. 17. 19. 21. 24. 26. 28. 30. 32. 34. 155,1. 3 . 5. 7. 9. 11. 13. 14. 15. 17. 19. 20. 22. 24. 26. 28. *30. 32. 34. 156,1. 2. 3 . 4. 5. 6. 8. 10. 11. 13. 15. 17. 19. 21. 23. 25. 27. 29. 31. 33. *35. 157, 1. 4. 6. 8. 10. 12. 14. 16. 18. 20. *22. 24. 26. *28. 30. 32. 34. 158, 3. 4. 6. 8. 12. 15. 17. 19. 20. 21. 23. 25. 29. 31. 33. 35. 37. $159, * 1.3 .5 .7 .9 .13 . *^{*} 15$. 19. 20. 22. 24. *26. 28. 30. 32. 34. 36. 160,1, 3. 5. 8. 11. 15. 17. 19. 25. 29. 31. 33. 161, 6. 7. 8. 13. 15. 17. 19. 23. 27. 29. 33. 35. 162, 3. 5. 7. 9. 11. 13. 17. *18. 28 ; schlossen $98,11.27 .118,29.128,18.151,16.153,16.155$, 18. 157,3 . 27. 161,6 . 8. 13. 17. 162,5 . 7. 13. 28 ; mahelschlo/s 127, 3. 18. $212,10.213,1$; schlo/sgattern 249,14 ; schlussel 47, 27. 69, 1. 97, 9. 98, 12. 99, 10. 110,14. 28. 117,29 . 119, 10. 127, 4. 128, 26. 131, 2. 3. *142, 22. 218,31 . 33. 219,2 . 233, 17. 250, 29. *257, 29 ; schlosser *96, 31. 97, 5. 17. 27. 31. 35. 98, 8. 12. 14. 18. 99, 3. 11. 14. 16. 18. 100, 25. 29. 32; schlossermeister 97, 12; abschlagen 68, 31; anschlagen 97, 28. 128, 3; aufschlagen 85, 22. 91, 7. 149,9 ; aufschlecht 98,33 ; beschlagen 72, 17. 99, 31. 33. 100,13; beschlahen 99, 30.100,1.3.14; verschlagen $* 215,25$; schlagen $45,13.87,17$. $81,1 . * 127,13 ;$ schlahen $50,10.65,10.99,17.101,20.128,24 ;$ geschlagen 65,11 ; anschlagen 97,33 . 101,27 ; schlecht $45,17.60,1$. 5. 6. 7. 8. 9. 11 . 12. 13. 14. 15. 17. 18. 61,1 . 2. 4. 6. 7. 8. 9. 10. 11. 12. 13. 14. 15. 16. 17. 17. 18. 19. 21. 22. 23. 29. 275, 19. 29 ; schlug 51,9 ; schlachglocken 109 , 28; schlachhaus 76, 21. 120,3; abschlahung 229, 33; afterschlag *74, 1. *3. *75, 26. *30. *32. *76, 32; durchschleg 288, 37; schlaher 56, 15; goltschlaher 174, 12; huntschlaher 103, 22. 105, 28. 112,1; messingschlahers 138, 32; peckschlaher 157,4; schlegel 164, 18. 20; Schlegel 100,5; schleift 112, 24 ; 
schleiffen 112, 32. 113,6; geschliffen 113, 2. 128, 24; durchschliffenden 248, 35; schleiffer 112, 20. 21. 24. 27. 33; schleufmewern 200, 28; schleiffmüll 113,6; schleiffreder 200, 24; schliffstein 113,4; schleiffin [schlaiffen, schleipfen] 130,10. 141, 25. 27. 33. 142, 1. 4. 6. 12. 17. *145, 29; abschleich 46, 26.130,13; schlai/sholtz 119, 8; schlott [schlett] *93, 21. 111, 25. 30. 32. 112, 4. 7. 17; schletten 111, 26; schlottfeger 111,23. 30. 112, 10. 16. *25; schlicht 59,32 ; geschlechter 71,$10 ;$ schlechtz $88,12.186,4 ;$ schlechten 99, 10. 106, 2; schlecht 110,21; ungeschlachtz 92, 19; Schleyer *133, 12. $*_{2}$ 7. *139, 15; Schleicher 56,21; Schlusselfelder 146, 15. 150, 24. 172, 19; zweite hand $30 \mathrm{sl}$ : beslossen 297,3 ; slo/s 296, 18. 20. 25. 30. 297, 1. 310,10; slossen 296, 25; felslo/s 298, 1 ; mahelslo/s 297, 2. 15. 24 ; slussel 296, 26. 326, 27; Slusselfelder 332, 29; sleipfen [sleyffen, slayffen] 296, 8. 328, 28. 329,15 ; slot $[$ slet $]$ 296, 20. 298, 22. 301, 23. 25; sleuch 307,17 ; aufslagen 303,1 ; aufgeslagen 297, 31. 301, 21 ; abgeslagen 331, 8; slahen 321, 21 ; slecht 322,37 ; geslagen 297,14 gegen 3 schl: schloss *307, 20; schlussel 308,9 ; schleifmül $* 310,10$.

c) Im mitteldeutschen sprachgebiet.

1. Urkundliche belege:

\$6. intschlichinde 1389, Cod. dipl. Sax. 2, 4. Meissner urkdb. no. 210; usgeslo/sen 1477 ib. no. 72 ; in das schlu/s 1448 ib. no. 73 : uzgeslossen 1423 ib. no. 75; anslan 1433 ib. no. 79 ; beslyssung 1457 ib. no. 115; geslecht, geslossen 1463 ib. no. 287; schlos, slos rechnung von 1477/78, ib. s. 96 ; geslechte 1506 ib. no. 328. - Slesia, slesisch heisst es nach Pietsch-Riickert, Entwurf s. 145 noch fast durchweg in schlesischen urkunden des 15. jh. 'doch begegnet Schlesia schon (l) 1428, Scriptor. rer. Siles. 6, 91 und 1434, ebd. 196.'

2. Belege aus literarischen denkmälern:

§ 7. Joh. von Frankenstein: 'öfters' schl (Ferd. Khull, Ueber die sprache des Johannes von Frankenstein. Progr. Graz 1880 s. 9). Buch von guter speise und J. Rothes Ritterspiegel: nur sl. - Stolle: stets sl gegen 1 schl; schlo/sen subst. s. 57. - Miscellanhs I. duod. 41 l schl: schlagen (vgl. Pietsch-Rückert, Entwurf s. 18). Luther: nur schl (das von Mönckeberg angefuhrte ratslahen ist nach Franke, Grundzüge s. $76 \S 89$ zu streichen).

\section{II. sm.}

1) In Oberdeutschland.

a) In Alemannien.

1. Urkundliche belege:

$\$$ 8. Glarus: Ruodolf den Smit 1289; von Smitten 1302 no. 33 ; Hug der smit 1320 no. 44; smitz (2) 1322 no. 46; Wernli Schmit 1411 no. 141; kupfersmit 1421 no. 167; golds chmid 4; smeltzen 1425 no. 175; Goltschmid 1425 no. 776; kupffer s chmid 1427 no. 180; Goldschmid (2), Hartman Schmid 1428 no. 182; Gold schmid (4) 1429 no. 185 ; Smerikon, Gold scmid (2) und Goldschmid (dieselbe person) 1437 no. 202; Goltsmit 1437 no. 207; Goldschmid 1440 no. 224; Goldsmit und Goldschmid (dieselbe person) 1440 no. 233. - Bàsel: Smidin 1348 no. 337, lat. nrk.; 
Johans der Smit 1356 no. 360 ; smilzet (2) 1363 no. 390 ; Smid s. 409, 6. a. 1371 , no. 414 , lat. urk.; Smidin (2) s. 437,35 u. 438, 6 a. 1375 no. 424 ; smid 1385 no. 458 , 8. 473,13; smacheit 1397 no. 502 , s. 558,30 ; sch millzet 1422 no. 628 , s. 736,9 ; Smitz 1430 no. 658 s. 780,26 . - St. Gallen: Goldschmit 1307 no. 1169; Goltscilmid 1312 no. 1216; des schmides 1347 no. 1441. - Vorarlberg: Schmid, Schmäklin 1452, Mone 10, 433. Hohenzollern: kloster Wald: Schmerlin 1350, Mone 10,477; Schmit 1356 ib. 483; kupferschmid, Schmid 1462, Mone 11, 113; Habsthal: schmalz 1394, Mone 11, 224. - Augsburg: aus der Smuter 1290 no. 124 ; zusmekke 1293 no. 193 ; chezzelsmid 1296 no. 160 ; smidhus 1325 no. 277; schmit 1345 no. 410; kesselsmits 1368, erster zunftbrief, no. 612; Hans der smit (ein kesselschmied) 1368, zweiter zunftbrief, no. 612; schmid (3) 1374 no.654. - Aus weistümern: smertzen (2) vor 1313, amt Eigen, Rochholtz s. 4 ; smeltzet 1351, Erlinsbach ib. s. 29 ; ger s chmid 14. jh. Ermatingen (Thurgau) Grimm Weist. 1, 239; schmaltz und smaltz anfang des 15. jh., hofrodel von Einsiedeln, Grimm 1, 151.

2. Aus literarischen denkmälern:

§ 9. a) Mainauer naturlehre: nur sm: gesmide s. 12 ; versmahtent s.14. - Maget krône: 5 sm: smack 4,9.14; 6,17; smaichen 3,63 ; versmêhel ${ }^{*} 4,337$ gegen 4 schm: schmacheit 4,201 ; verschmâcht ${ }^{*} 5,79$; schmecken 5,330; schmarck 11,13. - Hugo von Montfort: schreiber A: nur sm: smertzen $1,27 .{ }^{*} 4,14 ;$ smügen 4,133; smiegen 5 , 306 ; unversmogen ${ }^{*} 4,169$; schreiber B: 11 sm: smertzen $14,44.26,16$. *43. $27, * 80$. $33, *^{*} 7$; smeh 14,11 ; unversmogen $* 16,22$. *33, 125 ; smel 21 , 27 ; smacht 28,20 ; gesmeltzet 28,545 gegen 2 schm: schmacht 15,67; schmaragden 28, 552; schreiber C sm: smertzen *38, 4. - K u c hime is ter: 2 schm: schmeckend s. 108; Goldschmid ') 8. 224 gegen 1 sm: gesmack 113. - Stretlinger chronik: $1 \mathrm{sm}$ : smerzen 907 gegen sonstiges schm (8); ungeschmack 19,11; schmerzen 28,11. 89, 21. 140, 7. 141, 18. 155,12 ; verschmacht 125,24 ; schmach 126,13 . - Ulrich von Richental: nur schm: goltschmid 32. 182. 215; schmach 62; schmachk' 81 ; schmack 81 ; schmecken 81 ; schmak 84 ; schmid 182. 215. Auch bei eigennamen: in den Schmerlinen hu/s 38. 48; Schmerliko 152; Schmolentzgi 136. 139. 158. 191. 207. 209.

B) Merswin, Elsässische predigten, Dankrotzheim, Erläuterung der regeldes hl. Angastin: nur sm.

r) A ugstourger stadtbuch: nur sm.-Wackernagel: schmach 29, 12 (s. Weinhold s. 7). - In gold: 1 sm: versmächen *75 gegen sonstig. schm (30): schmachayt 3. 4. 32, 1. 2. 21 ; geschmeyd 6. (2) 7. 8; verschmachen ${ }^{*} 12 .{ }^{* 32}$. $\left({ }^{*} 2\right) .{ }^{*} 35$; verschmachlich ${ }^{*} 16$; geschmacht 17 ; schmutz 17 ; verschmach ${ }^{*} 28$; schmak 29.73 ; schmidet 29 ; schmid 39 . 78 ; fürschmeckender ${ }^{*} 40$; verschmachl ${ }^{*} 45 .{ }^{*} 65 .{ }^{*} 68$; schmecken 49 ; verschmächung ${ }^{*} 53$; schmiten 78 ; schmotzot 80 . - Conrad von Weinsberg: 1 sm: smyde 41 gegen stetes schm im worte schmid, das fast auf jeder seite vorkommt. - Hermann von Sachsenheim, Mörin: $18 \mathrm{sm}$ :

1) So liest Z, aber V (die jüngere hs.) hat Goldsmid. 
smiert ${ }^{*} 154 ;$ smitlen 285 ; ungesmiert $368 ;$ smag 432 ; smidknecht 551 ; versmahen $* 658$; versmacht $* 1062$; smoezl 1571 ; smach $* 1632$; kupffersmid *1952; smehet 2514. 2525; smal 3032 ; gesmakl 3374 ; versmaucht *3540; smach 3714; gesmecht 5234. 5560 gegen 12 schm: schmal 115; verschmit ${ }^{*} 287$; schmeher 1805; schmog 1839; geschmecht 3843; schmicz 4229. 4231; schmakst 4440; schmor 4663; schmid 4903; schmoczl 5805; schmerz 5996. - Der goldene tempel und Jesus der arzt: nur schm: a) schmal 866 ; verschmacht $* 1281$; - b) verschmach *99; schmal 121, 135. - Kaufinger: nur schm (23): schmächlich 1, 327; verschmacht $* 2,31$; schmackes 3,551 ; schmachlichen 3,665 ; schmuckt 3,693 . 15,69 ; schmielen 4,185. 195; schmal 5,132. 9, 216. 16, 725; schmachlich 7, 190. 13, 45; verschmolzen *9,107; schmukt 11,83 ; schmerzen $11,103$. 144. 14,449; schmachait 11,271. 12,183; schmack 13, 56. 61 ; schmach 14,673 .

b) Im bairisch-österreichischen sprachgebiet.

1. Urkundliche belege:

$\$$ 10. Jordan der Smide 1311, Urkdb. d. l. o. d. E. 5 no. 44; Chunrat Smuchenpfeninch 1322 ib. 5 s. 322 no. 335 ; Goltsmid 1327 ib. 5 s. 492 no. 498 ; smit 1332 ib. 6 s. 59 no. 43 ; Ertsmit 1334 ib. 6 s. 114 no. 106; Smit, Chaltsmid 1361 ib. 8 no. 29 ; Smid 1363 ib. 8 no. 120; pei der Smelczer stainhauffen 1367 ib. 8 no. 322 ; Stephan der Smyd 1369 ib. 8 s. $406_{3}$ no. 411 . - Aus weistümern: schmaltz, ge schmelert 14. jh., Pillersee T. w. 1,90; schmer, schmelert 1405, Nonnberg bei Salzburg ö. w. 1, i10; Friedrich der Smicher (2) 1411, Trins T. w. 1, 292; goltsmid 1434, Brandenberg T. w. 1, 135; smalz 15. jh., Innsbruck 'T. w. 1, 231; schmach 1487, Zillerthal Ö. w. 1, 317; Lienhard Smid 1497 und 98, Mittersill 0 . w. 1, $283 \mathrm{ff}$.

2. Belege aus literarischen denkmälern:

§ 11. Oswald von Wolkenstein: 77 (76) sm: smal 1, 6, 2. 3, 1, 32 ; smecken $* 4,3,6$; smale ib. $* 3,13 ;$ smutz $\left.^{1}\right) 5,1,56$; gesmissen ib. 1, 79; ungesmach $^{2}$ ) 6,93 ; smerlz $* 7,3,11 ;$ smer $14,4,17$; smalen $16,2,5$; smucken ib. 3,16 ; smiegen ib.; goltsmid 17, 3, 24; smal 17, 2, 4. 31; 18, 8, 1; smieren 18, 2, *2; smetzen $24,2,7$; gesmech $25,4,3$; smucken 29,1 , 34 ; smirbt 30, 1, 15; versmogen ib. 1 , *9; smutz ib. 3 , 9; smucken 32 , 1,10 ; smelhlein $35,2,15$; smucken $35,3,37$; smerlzen $36,3,10$; smach ib. 6,3 ; smutz $39,1,26$; smal ib. 2,24 ; smertzen $44,3,2$; smutz 45, 17; smel $47,3,6$; smuck 48, 1, 11 ; smutz 49, 2, 6; smal 50, 2, 6; smertzen $51,3,19$; smiel $52,1,3$; smal ib. 1, 6; smutz ib. 1, 12; gesmogen 63, 3, 14 ; smuckt $65,3, * 5$; smel $66,2,10 ;$ smutz $67,2, * 11$; smirb ib. 3 , 12 ; versmächt ib. $4,{ }^{*} 11$; versmahen $68,1, *_{12}$; unversmacht $69,1, *_{7}$; smortz $70,1,30 ;$ smeug $71,1,8 ;$ smielich $72,1,8 ;$ smuck 76, 1, *85; smertz $77,4,10 ; 78,1,5 ;$ smal $79,3,5$; smertz $88,2,7$; gesmuckt 90 , 2,9 ; smucken $93,2,6$; smercz $94,2, * 11$; smäch $95,4,5$; smecken ib.

1) X liest schmatz.

2) I liest ungeschmag. 
5, 2; versmahen $106,7, *_{10}$; smechlich ib. 8,6 ; smertzen ib. 9,$2 ;$ smach $107,1, *_{14}$; smutz ib. $1, * 19$; smuck $108,6, * 16$; gesmitt $109,4,14$; versmacht $110,2,{ }^{*} 3$; smertzen ib. $3,{ }^{*} 4$; versmitt ib. $3,{ }^{*} 6$; smecken $111,2,8$; smilzet ib. 2, 18; smerlzen 116, 2, 15; smecken 118, 6, 4; smertz 119, 1, *17; versmach $121, *_{112}$; [versmach $122,4, *_{5}$ nur in W] gegen 10 (8) schmatz [nur X] 5, 1, 56; ungeschmag [nur I] 6, 93; schmilzen $\left.{ }^{1}\right) 29,2,32$; schmertzen $33,1,8$; schmertz $37,1, * 3$; schmucken $64,1,6$; schmatz $73,2,2$; schmertze $87,2,5$; schmertz $117,4,1$; schmertzen 119, 1, 3. - Schiltberger: $4^{2}$ ) sm: Stephan Smicher 6, 11; smach 32, 23 ; gesmach 80,21 ; smahe $* 111,5$ gegen 3 schm: geschmack 79,3 ; schmähen 88,4 ; schmach 98,30 . - Tucher: erste hand: 2 i sm: smide 96, 31 ; smits 99,26 ; smiden 242,20 ; negwersmit $* 162,19$; schersmit $* 101,5$; plechsmit 190, 20; klingensmide 270, 24; Smitgassen *190, 19. 285, 33; Pfandsmitgassen 225, 16. 226, 1. 10. 234,10; smeltzhütt 270, 26; smeltzhutten $*_{144}, 16 .{ }^{*} 156,21 .{ }^{*} 162,24 . *^{*} 11,25 .{ }^{*} 219,24 .{ }^{*} 222,19 ;$ smeltzt 270 , 25 ; versmeltzen ${ }^{*} 253,33$; smiren 249,13 ; gesmiret 249,16 ; wagensmire 116, 28; smaltz 270, 26; sweinensmaltz 249,12 gegen 62 schm : schmiden *99, 2 ; $\operatorname{schmid}(t) 41,23.97,5.18$. 25. 27. 99, 5. *6. 18. 99, 2. 21. 25. *100, 23. 101, 12. 124, 12. 128, 3. *159, 1. 9; schmide 41, 15; der schmiten *41,18; schmitten 84,17 ; goltschmit 84, 17, 146, 30. 155, 20. 156, 5. 31. 158, 6. 242, 1 ; klingenschmit 161, 21. 268, 36. 269, 5. 9; Pfantschmit 136, 24; Pfantschmit(d)gassen 136, 25. 137, 24. 36. 138, 3. 194, 5. 225, 17. 236, 2; Penlnschmidt 49,7 ; plechschmit 154,23 . 285,31 ; rotschmit $157,12.160,11$; Schmit 156 , 19. 158, 38. 209, 26 ; Schmidt*101, 13; Schmidmar 138, 26. *226, 26; schmiren 248, 21; wagenschmir 116, 16. 19. 27. 292, 29; schmeltzhutten *156, 23. 269,1 ; schmaltzkessel *288, 29; schmekt 115,11 . 239, 2. 2; schmeket 236, 16; zweite hand nur sm: Pfansmitga/s 294, 14. 295, 2; klingensmide 313, 28. - Beschreibung einer seereise: nur sm: smirt z. 11; smyd z. 40. - Nürnberger polizeiverordnungen (13. u. 14. jh.): nur sm.

II. Im mitteldeutschen sprachgebiet.

1. Urkandliche belege:

$\S$ 12. schmalz 1287, Henneb. urkd. 3, 1550 (vgl. Weinhold, Mhd. gr. $\left.{ }^{2} \$ 208\right)$; Petir Smyd 1401, Meissner urkdb. St. Afra no. 336 ; Malhis Mezzersmid 1444, ib. no. 273; Mattes Smid 1453, ib. no. 279; Han/s Smidigen, vorsmirt 1477, ib. schlossbaurechnung 8. 95; sch mide (2) 1477/78, ib. schlossbaurechnung 8. 97 ; smiren 1479/80, schlossbaurechnung ib. s. 97; Matys Smyd 1480, ib. no. 142.

2. Belege ans literarischendenkmälern:

$\S$ 13. Job. von Frankenstein, das Buch vonguter speise, die übersetzung der Offenbarung Johannis, Rothes Ritterspiegel: nur sm. - Miscollanhs: auch schm: schmerczin 1, 40; vorschmehunge 1, 150 (Pietsch-Rückert, Entwurf s. 144).

1) Weber gibt irrtïmlicher weise im glossar smitzen an.

2) Nicht gerechnet sind versmahen $\mathrm{DH} * 91,15$ und smainst 84,7 . 


\section{III. sn.}

I. In Oberdeutschland.

a) In Alemannien.

1. Urkundliche belege:

§ 14. Glarus: abgeschnitlen 1438 no. 218. - Basel: in dem Snatt; des Sniders 1360 no. 380 ; schne (2) 1363 no. 390; Snider 1371, s. 416, 2i; Riddolf Schnider 1375, s. 438, 23 no. 429; Riddi Schnider 1390, no. 475; Schnider 1391, lat. urk. s. 509, 8 no. 477; snider 1400, 8. 599, 3 no. 529 ; schne 1422 , s. 736,9 no. 628 ; Claus Schniders ib. 737 (ઉ); schnider 1424, s. 768, 32 no.645; Henmans Schnewlin 8. 812, 17; Snewlin 813,17; Heini Snider s. 812,18, 1436, no. 682; Heini Snider 1437, s. 816, 37 no. 685; Henny Snider 1437, no. 687 s. 818, 30; Schnider 1442 no. 716; Schnider 1444, s. 854, 25 no. 720. - St. Gallen: In schnail 1302, Wartmann 3, s. 319; ze sneita, (derselbe ort) 1304 ib. 336; snewisse (3) 1320 no. 1259 ; âhschnitter 1342 no. 407 . - Vorarlberg: schneberg 1548, Mone 10, 430; Schnider 1452 ib. 433). - Königsbrunn: (Mone 10, $120 \mathrm{ff}$.): schnabel, snabel, schnir 1425. - Villingen: schnod, schnell 1459, Mone 8, 476. - Augsburg: Snegalle 1283 no. 76; Wernher der Snelman 1284 no. 88; Wernher Snelman 1290 no. 124; Snellen 1299 no. 174 ; zerschnittens 1368 no. 612 ; schnider 1374 no. 659 . - A u s weistümern: sniden vor 1313, Eigen, Rochboltz s. 4; die snursleipfe ca. 1322, Elfingen ib. s. 9; sne, Ulli Schnider 1351, Erlinsbach, ib. s. 29 und 34; abschniden mitte d. 14. jh., Ermatingen (Thurgau), Grimm 1, 239; Rudi Snider 1412, Bersikon, ib. 1, 49; snur 1417 Winkel (Zürich), ib.

1. Belege aus literarischen denkmälern:

\$15. a) Mainauer naturlehre: sn: sne s. 14. - Basler Nibelungenbruchstücke: schn: schnelle 1362, 3. 1367, 2. *1371, 4 . ${ }^{*} 1643,1$. - Der uaget krône: schn: schne 2, 12. *5, 268. 5, 305; schneiden $* 5,488$; geschneit 5 , 268. - Hu go von M on tf ort: schreiber A: 8 sn: sne ${ }^{*} 2,35$; sneller 4,11; snell 4, *124. 164. 5, 58. 60. 63. 226; gegen 1 schn: verschneiden *5, 8; schreiber B: 1 sn: snell ${ }^{*} 27,38$ gegen 16 schn: verschneiden ${ }^{*} 15,41 .{ }^{*} 26,35 .{ }^{*} 60$; abschniden 28,110 ; beschniden 28,43 ; schnell ${ }^{*} 15,45 .{ }^{*} 28,41.33,25 .{ }^{*} 5$; schne $15,141 .{ }^{* 19}, 31.27,16$; schnöde 24, 130; schnöd 28, 432. 652; schnuck 31, 145; schreiber C: schn: schnuore 38,116 . - Kuchimeister: nur schn: schnaid s. 41; angeschnitten 42; Schneggenburg 138; schne 242. 244; Schnabelburg 316. Stretlinger chronik: nur schn: schnell 6,11; abgeschnitten 100,28; abschnitte 155,13; schnellen 162,3. - Richental: nur schn: schnider *32. 182. 183 ; fürschnider *58; verschnitlent $*_{120}$; schnetzly $*_{40}$; schne $6+$ (2); schnilzt 120; schnüren 139.

B) Merswin: nur sn: snede $33(2)$; 120.123 ; snident 53 ; sniden 129. 136. 139. - Elsässische predigten: nur sn. - Dankrotzheim: nur sn: besnilten v. 43 ; snee 61.82 ; snider $482 .-$ Regel des hl. A ugustin: nur $s n$ : sneiden 51 a.

r) Augsburger stadtbuch: nur sn. - Wackernagel: $s n$ in no. 42-52 (vgl. Weinhold 10), seltener schn: schniden 35, 58; geschnilen 
28,31 (vgl. Weinhold 7). - Inguld: nur schn: abschnitend 19; schnelikait 23; schnellen ib.; schnegg 29; schneidet 32; schneider, virschneider *40; schnell *41; schnöd, schnöden 44; schnell *45; glokschnier 62; schnöden 63 ; schneidend 66 ; schneiden 67 ; schnateren $* 68$; schnallen. schnatertafel, schnuer 68 ; verschneident $* i 1$; schnell 75 ; schneit, erabschneyderin 51 . Ruland: schn: gwandschneider 18. - Conrad von Weinsberg: nur schn: schnyder (6) n. pr.; schnürlin 4; abzuschniden 18. - Hermann von Sachsenheim, Mörin: 22 sn: snabel 401. 548. 685; snoder 539; snöd 580 ; snöden 981. 1089. 1537. 3276; snödi ${ }^{*} 65 \bar{i}$; sneller 881.3158 . 3450; snell *1054. *2452. *2870. 4021. 4038. 5648; snöder 2904; snucor *3246; snöde 3487 gegen 19 schn: schnabel ${ }^{* 250}$; schnell 284 . $3687 . * 4561$. *5346. 5372. 5937. 5947. *5980; schnitten 710 ; schnoder 1643 ; beschniden 1991; schnöden 2003. 4798; schnecken ${ }^{1}$ ) 2097; schnede *2683; schnitt (subst.) 2882; schnellen 4227; schnode 5898. - Der gold en e tempel: nur schn: schnuor 67. - Jesus der arzt: nur schn: schniderstuol 75. Ka u f ringer: nur schn: schnelle 2, 82; schnell 3, 232. 16, 451. 111; schnewei/sen 4, 192. 9, 212 ; schnelliclich 12, 242; schnaid 13, 239. 14, 278. 732; schnäll 13, 403. 563 ; abgeschnitten 14, 295; schnuren 16,123; schnöd 16, 358. 485. - Reimchronik des Joh. Kurtz: nur schn: schnell v. 33. 72. 572; schnödikait 351; schneggen 910.

b) Im bairisch-österreichischen sprachgebiet.

1. Urkundliche belege:

$\$$ 16. Wernhart der Snöde 1321, Urkb. d. l. o. d. E. s. 290 no. 302; snür 1330 ib. 572 no. 575 ; Reicher der Sneider 1344 ib. 6, 467; C'hunrals dez Sneider 1359 ib. 7,625 no. 615; Görig der Sneider 1362 ib. 8, 61 no. 59; Peter Snabel 1365 ib. 211 no. 208; snell (2) 1366 ib. 278 no. 281. - Ans weistümern: sne bergrechte in der Gastein und Rauris I (1300-1350), 0̈. w. 1, 196 ff.; Schneider (2) 1398, 'T. w. 1, 294 ff. Pfons; sneibl (2), aufsneiden Innsbruck 15. jhd., T. w. 1, 231; schneflü/sl 24, 3; abschnitt 25, 43 Oberinntal, Flaurling 15. jhd., 'T.w. 2, 23; schnee St. Valentin auf der haid, 1489, T. w. 2, 352.

2. Belegeaus literarischen denkmälern:

$\S 17$. 0swald von Wolkenstein: 60 sn: snir $1,4, *^{*} 19$; snee ib. 5,16 ; snell $2,1,{ }^{*} 14 ;$ snellem ib. 1,20; gesnúret $2,3,11:$ snee $3,1,25$; snellen $4,1,27$; gesnitten $6,1,12$; sniir $12,4,{ }_{4}^{*}$; snellet $13,5,8$; versniet ib. $6,{ }^{*}$; snarcht $13,10,{ }^{*} 3$; snee $16,1,9$; snabel $18,3,{ }^{*} 6$; sneid $\mathrm{ib} .8$, ${ }^{*} 6$; snöden $24,2,7$; versnürt $25,3,{ }^{*} 16$; snödem ib. 4,3 ; sneyden $\mathrm{ib.} 4$, $* 18$; versnait $26,{ }^{*} 150$; snódes $29,1,30 ;$ snödem ib. 2,22 ; snees $30,1,8$; snüren ib. 2,36 ; snattert $31,4,40 ;$ snee $33,1,{ }^{*} ; 35,1,42 ;$ sneblein 37 , 1,$5 ;$ snell 40,1,8. 44,3,18; snäggel 45, 20; snell 57, 3, 4; snäggl 58, 1,1; snurra ib. 4,5 ; godersnal $60,3,{ }^{*} 8$; snell $67,2,8$; versnorpffen $70,3,{ }^{*} 9$; gesneude ib. 3,19 ; snurlin $71,2,5 ;$ gesneud ib. 3,4 ; snee $76,3, * 31$; snee $75,1,{ }^{*} 15 ;$ snell $88,2,15$; sne $90,1,{ }^{*} 2$; snir $91,2,{ }^{*}$; snöden $98,2,{ }^{*}$; snurr 106, 11, *7; ubersnellet 108, $3,{ }^{*} 10 ;$ snee $109,2,{ }^{*} 3$; snell 112, $2,13$.

1) So mit Martin; die handschrift hat schnken. 
$115,5,12 ;$ snöder $117,5,10 ;$ snallet $\mathrm{ib.}{ }^{*} 6,8$; snöde $118,1,1 ;$ snee $\mathrm{ib} .4$, ${ }^{*} 12$; snöden $119,2,16$; besnaid 121,2 ; snell ib. 13,187. 122,1,3; besniten 118, 1, 1 gegen 2 schn: schneller 103,1,11; beschnaid 122,1,1. - Schiltberger: 2 sn: snecken $* 45,11 ;$ besneyden 86,21 gegen 11 schn: schneill 48,8 ; schneyll 94,19 ; schneyden 52,14 . 62,8 . 92, 7 ; schnabell 69,28 . 70, 25 ; beschneyden $86,21.22 .94,12 ;$ schneydens dingk $\left.87,6 .{ }^{1}\right)-\mathrm{Besch}$ re i bung einer seereise: 1 sn: srur 61,11 gegen 2 schn: schnyder z. 41; schneyt 7. 291. - Nürnberger polizeiordnungen (13. u. 14. jh.): nur sn. - Tucher: erste hand 34 sn: snur 110, 4; snur 264, *15. 15; snürlein 236, 2i; rebsnior 111,16; snurmacher 158, 31; Snürer 95, 3; snurlechtz 166, 12; gesnitten 241,16; snehe 252, 24, snee 252, 26. *26. 253, 28. 32. 32. *36. 254, 4; Snödin*165, 32. *166, 5. *13. *32. 178, *15. *24. *25. 179, *7. 210,*29. 31. *217, 21. *218, 10.*222, 3. *270, 18; Snaltzer 156, 8; Snitzers 172, 9; Snigling 205, 13 gegen 60 schn: schnur 263, т; rötelschnür 110, 3 ; schneiden 72, 25. 76, 18. 79, 19. 112, 23. 26. 32. 116, 7. 120,19. 24. 121, 14. 19. 28. 122, 32. 33 ; schniden 121,26 ; schneit $\lfloor d t]$ 72, 23. 112,34. 120, 25 . 35 ; schneidet 121, 5. 13; geschnitten 75, 33. 78, 8. 20. 120, 20. 121,1; au/sgeschnitlen 123,1. 269, 7; schnit 112, 32. 34. 120, 26. 28. 34. 121,1.20. 22. 123, 10. 292,30 ; schneider 151, 9. *34. *152. 156, *8. *25. 160, 23. 161, 27. *162, 18; schneiders 146,13. 151,32; Schneider 209, 32; schne *234, 24; Schnödin 206,*4. *i; schneller 130,36. 131, 21. *210, 23; schnellern 109, 28. 215, 24; störchschnabel 100,11; zweite hand 4 sn: sneller 296,14. 15. 16. 298, 27 gegen 16 schn: schneiden $317,{ }^{*} 14.14 .322,30$. 35. 36. 323, 2 ; geschneiden 323,15 ; schnaid (schnait) 304, 10. 323, *9. *10; geschnitten 305, 37. 322, 27; au/'sgeschnitten $317,19.325,2$; schnit 322,26 . 323, 9 .

II. Im mitteldeutschen sprachgebiet.

1. Urkundliche belege:

$\S 18$. sneidewerk 1277, Nassauer urkdb. no. 930; in Sneilhaue 1280, ib. no. 974; Alheid Snellens 1300, ib. no. 1300. Hans Snyder 1418, Meissn. u. no.73; Michel Snider 1477, schlossbaurechnung ib. 95; Michel Schneider (2) schlossbaurechnung von 1477-78, ib. 96; Caspar Sneider 1480, ib. no. 180 .

2. Belege aus literarischen denkmälern:

$\S 19$. Johannes von Frankenstein, das Buch vonguter speise, übersetzung der Offenbarung Johannis, Stolle haben nur sn. - Miscellanhs. v. 1440 schn in schnaucze (vgl. Pietsch-Rlickert, Entwurf 144).

\section{IV. sw.}

1) In Oberdentschland.

a) In alemannischen sprachgebiet.

1. Urkundliche belege: ${ }^{2}$ )

1) DH bieten noch: schnitt 91,3; abschniden 91,8; schniden 92, 7.

2) Hierbei lasse ich die so häufigen pronomina und pronominaladverbia: swer, swaz, swie, swenne etc. einstweilen bei seite. - Ueber diese wörter und ihr sporadisches auftreten mit sch s. weiter unten. 
§ 20. Glarus: swande, swclen 1259 no. 31 ; geschwornen (2); swuoren (2), geswesteran, swester, swander 1302 no. 33; Swilz (ö) 1316 no. 38; Schwyz 1316 no. 39; Switz (ï) 1318 no. 4l, 1319 no. 42 ; swesler, swantz, besweren 1322 no. 46 ; swestern 1322 no. 47: gesworn, Switz 1323 no. 49; swester 1333 no. 56, 1340 no. 59 (ö); Schwilz 1350 no. 66 ; Switz (0); gesworn (ï) 1352 no. 69 ; gesworn (3), sweren (adj.), verswigen 1359 no. 76 ; gesworn 1367 no. 80 ; swöstere, schwagere 1370 no. 86 ; gesworen (2), Switz, sweren, geswistergit 1387 no. 105 ; geswuoren (2), geswornen 1393 no. 121; gesweret, geschworn, geswornen 1395 no. 126 ; gesworn (ö), swerrin 1400 no. 131; swern 1402 no. 132: gesworn 1406 no. 137; Swilz, Heinrich Swander, Swartz 140־ no. 138; gesworn, geswornen (ö), swerren (2) 1408 no. 139; swanden, gesworn (2) 1411 no. 141; Schwandegg, schwin (2) 1412 no. 144; Swilz 1415 no. 155; swerren, geswecht, geswornen 1419 no. 159; Swilz 1419 no. 160; gesworn (2), swerren (2) ib.; gesworn 1419 no. 162; Swilz, Swanden 1419 no. $170 ; \operatorname{Swilz~(ö)~} 1421$ no. 166; Swilz (s. o.); gesworn (s. 0.) 1421 no. 167; Swilz (ن̈) 1421 no. 168; geswornen 1422 no. 169; gesworen, swerend 1424 no. 173; Switz (ö), geswornen (3), swerren (2), swechern, geswechert 1425 no. 175; Swytz (s. o.), sweren 1425 no. 176; geswornen 1425 no. 195; swilz (2), gesworen, sweren 1428 no. 182; Swilz, gesworen (2) 1420 no. 185; swager (s. 0.), swöster 1429 no. 186: Swilz (ï), gesworn neben geschworn, swerren neben schwerren (3) 1437 no. 201; Swilz (ö), gesworn (ï), geschworen, schwere (verb.), swerem 1437 no. 202); schwager, schwösler, geschworn, Schwytz (2), Swytz [Switz] (3) 1437 no. 207; swager, swär, swärer, swerrint. Switz (2) 1437 no. 209; Swilz (2) 1437 no. 212; swesler (2), Swylz (ö) 1438 no. 216; Mälischwand, Schwilz (8. 0.), beschwät (3), geschwornen (4) neben geswornen (4) 1418 no. 218; Swylz (ن̈), gesch worn 1439 no. 220; Swilz (ï) 1439 no. 221; Switz 1440 no. 224. 225. 226; Swylz, swerren (2), gesworn (2), geswornen 1440 no. 22т; Swytz (s. 0.), geswornen (o), gesworn, gesworner, beswärung (o) 1440 no. 2:3. - Basel: swester 1319 no. 251; gesworn, swin 1324 no. 272 ; swester, geswistergide 1332 no. 293; swester 1334 no. 298; swester (2) 1337 no. 303: geschworen 1342 no. 321 ; geswornem 1343 no. 322 ; 134 i no. 332 ; geswornen 1348 no. 338 ; swerer 1350 no. 344 ; 1353 no. 352 ; sweren, gesworn 1355 no. 3509 ; swager $135 \check{6}$ no. 360 ; swerer (2), gesworn 1356 no. 364 ; swach, swester 1358 no. 369 ; swerer, gesworne (2), swerent, geswerent 1359 no. 377; Swaben 1360 no. 379; Norlswaben (2) 1360 no. 380 ; swert verb., swuir, swerer 1360 no. 381 ; geswechert, gesworn, swern verb. (2) no. 383 ; schweren 1363 no. 389 ; sweren no. 391 ; Swaben no. 393 ; swerer 1365 no. 399 ; gesworn (4), geschworn 1366 no. 400 ; swesler (2) 1369 no. 404; swerer (3) 1371 no. 412; swingelt (2), swesler 1371 no. 415 ; swester (2), geswistern, swier 1371 no. 416; swager no. 417; swerer (2) 1372 no. $\left.419^{1}\right)$; swerer 1373 no. 421 ; Swaben 1374 no. 428 ; swester $13 i 7$ no. 433; geswisterigen 1382 no. 449 ; swester, geswistergyd (o) 1354 no. 455 ; geswornem (2) 1385 no. 458; swerer no. 461; swerer (3), swerent,

2) Hier sei auch Oswalt ib. 422, 22 erwähnt. 
gesworen 1386 no. 463 ; swinger 1388 no. 463; swerer, gesworn no. 469: swester (2) 1390 no. 474 ; no. 475 ; swingeltes 1392 no. 482 ; swerer no. 484 ; swerer, Swobes, Swabes (2) no. 486; swere ${ }^{1}$ ), gesworn ${ }^{1)}$ (2) 1393 no. 489 ; swerer 1395 no. 494; swerer (2), gesworn (2) no. 496; swester 1396 no. 497; swester, sweslern (2) no. 498; geswornen, gesworn, Swarlz no. 500; gesworn, swechert 1397 no. 502; volswerer 1399 no. 509; swerer (3), gesworu (2) no. 519 ; swerer (3) no. 520; swerlichen 1400 no. 526; geswornen (2), sweren verb., ze swere 1406 no. 548 ; swin, swerer, gesworn no. 550 ; geswornen 1411 no. 575 ; gesworn 1416 no. 592 ; beswern, gesworen no. 595 ; gesworn 1417 no. 601 ; swin (2) no. 603; geswornen (0) 1418 no. 606; sweren 1420 no. 616; gesworn, Swarlzenburg (2) 1422 no. 627; geswornen (s. o.), geswistrides, sweren verb., no. 628; Swob, Swartzenburg no. 629; ges čhwornen (5) no. 630 ; sweren no. 631 ; swellen subst., no. 637 ; geswornen (3) no. 639 ; geswornen (o) 1423 no. 640 ; gisworen 1425 no. 646; swingelt 1430 no. 660 ; swerer 1432 no. 670; ges chworen (0) 14.35 no. 674; gesworn no. 678 ; 1436 no. 682 ; schweren verb. 1437 no. 686 ; gesworne no. 687; Swop, Hans Swebili no. 688; geswechert 1438 no. 689; sweher, sweren verb. no. 692; geschworn 1439 no. 699; gesworn, swerer 1440 no. 707; schwechert no. 708; swester 1442 no. 711 ; swine (2), gesworn 1445 no. 722. - St. Gallen: Swarzinstein 1307 no. 1169 ; swester (2) 1315 no. 1225 ; swager, swester no. 1229; Swigger no. 1235; Swarzenbach, Swiger 1319 no. 1249 ; geswistern no. 1250 ; swagern (2), swager (3), swesterman, swester 1320 no. 1259; beswerin no. 1261; Swiger (ï) no. 1262; swester no. 1263 ; 1321 no. 1272 ; swir no. 1275 ; sweret, gesworn (2) no. 1276; gesworn no. 1279 ; swester 1322 no. 1285 ; Swegler no. 1289; swuoren, gesworen 1324 no. 1296; gesworen, swerren 1327 no. 1314; Swigger no. 1318; swester no. 1319 ; gesworn 1328 no. 1322; geswistergide, gesworn 1329 no. 1332; sweslcr (ï) 1330 no. 1335 ; besweren beschweren 1335 no. 1361; gesworn 1337 no. 1377 ; 1340 no. 1395 ; geswornen 1342 no. 1403 ; swesler 1344 no. 1416 ; gesworn no. 1421 ; geswecht no. 1422 ; Swaben, Swanningen 1345 no. 1431; gesworn, Swenningen 1346 no. 1439 ; sweren, gesworn 1347 no. 1447; beswären 1348 no. 1456 ; gesworn, geswecht 1351 no. 1476 ; swestren, Swaigalp 1353 no. 1497. - Vorarlberg: swäher, ge schwornem 1448 Mone 10, 430; gesworen, geswornen, swager, schwigen 1452 ib. 433. - Kloster Wald (Hohenzollern): swesler (2) 1311 Mone 10, 454; 1331 ib. 464 ; (0) 1333 ib. 467; Swigger (2), swehers 1334 ib. 468; Swigger 1338 ib. 473 ; swester (s. o.) 1342 ib. 473 ; swuor 1345 ib. 475 ; swester (2) 1350 ib. 476 ; Swiggcrs 1359 ib. 483 ; swester (s. o.) ib. 484. 485; swester (3). swestran, swestren, swechsten (2) 1388 Mone 11, 88); schwehers (3), schweher 1392 ib. 92; Schwaindorf 1397 ib. 97; swester (2) 1408 ib. 102 ; swöster ib. 104; swöstran 1411 ib. 105; Swaindorf (2) 1438 ib. 108; sch westermun, schwäger, sch wiger 1467 ib.115. - A u g \& b u rg: swestergun 1301 no. 181; Swaben 1312 บu. 221. 222. 223; swesler 1316 no. 242 ; swigger 1317 no. 248; swesler, gesworn ib.; Hainrich der Schwap 1318

1) So lesen beide exemplare der urkunde. 
no. 248; swaber, swigger 1319 no. 257; geswie (subst.) 1320 no. 259; swange 1322 no. 263; swester, geswistergid 1324 no. 270, swester, swager 1327 no. 284; beschwoeret, swar adj. 1329 no. 290; swester, swager 1330 no. 292, 294; swester (ï) 1330 no. 297; Swaben, gesworen, sweren swerent 1330 no. 299 ; gesworen (ï) 1331 no. 302, 311; beswort, swaren adj. 1332 no. 316; Swauben, gesworen, sweren 1333 no. 324; geswestergen 1333 no. 325 ; swaulmule 1334 no. 331; Swaben 1336 no. 339; swester no. 343 ; gesworner 1337 no. 348 ; swalmullner 1338 no. 354 ; sweher, swager 1339 no. 362; swecher s., swüren no. 366 ; beswären 1340 no. 368 ; sweher 1343 no. 398, 1345 no. 408; geswester 1345 no. 410 ; gesworen 1348 no. 437; besweren, swere adj. 1348 no. 448 ; swester (ö), geswester no. 450 ; Swawen no. 454; Swoben no. 455; beswerung 1349 no. 462; Swigger, besweren, gesworn no. 468; swin no. 472; swester 1350 no. 479 ; beswaren 1351 no. 485: gesworn (ï) 1352 no. 492 ; gesworen, swerlichen 1353 no. 494 u. s. f. sw. - A u s weistümern: swerel vor 1313, Eigen, Rochholtz s. 4; Swartzenbrunnen ca. 1322, Elfingen ib. 9; swerren, gesworen 1348, Berkon ib. 19 ; geswornen (2) 30; swin 32 (2) 1351, Erlinebach ib.; Schwinton (2) 1363 , Birmenstorff ib. 46; schwerren, schweren, schwartz, schwantz mitte des 14. jhd., Ermatingen (Thurgau) Gr.W.1, 239; Schwend, schwenden (2) ca. 1408, Rudolfstetten Rochholtz s. 59); Philipp Swerler geswistegit neben geschwistergit, swinen 1412, Bersikon Gr. W. 1,49; swantz, swerte 1417 Winkel [Zïrich] ib. 86. - swere anf. d. 14. jh., Heinesbrunn Gr. W. 4, 93; gesworen, sweren, swester (2), schwager anf. d. 14. jh., Altenschwiler ib. 9 u. 11; schwin 1382 [?], Niederburnhaupt ib. 75; verswigen, sweighoffe, schwecher ausgang d. 14. jhd., Riespach, ib. 3 ff.; ge schworen, schwacher (2) 1420, Preutzingen ib. 9; ge sch worner; schwacher, schwachr 1420, Werenthausen ib. $1 \mathrm{ff}$.

2. Belege aus literarischen denkmälern:

§ 21. a) Mainauer naturlehre: nur sw: swarzir 1; swinde 2. *3; swerer 3; swendet 9 . - Maget krône: 7 sw: swebet 3,101 ; gesworn 3,130 ; swert $4,244.526$; swuer 4,273 ; sweb 5, 367; verswant $*_{5}$, 328 gegen 8 schw: beschwere 2,48 ; geschwind $3,8.4,144$; schwur 4,374 ; schwestern 5,211 ; schwester $5, * 185$. 324 ; schwarz 5,45 . - Basler Nibelungen bruchstücke: nur $s w$, vgl. Wackernagel a. a. o. - Hugo von Montfort: schreiber A $s w$ : verswigen $3,{ }^{*} 53$; swer 8 . ib. 68 ; swartze 5,59 ; swuren ib. 102 ; swer adj. ${ }^{*} 5,129$; swigen 9,6 ; schreiber B $s w$ : swebt 12,15 ; swer v. ${ }^{*} 13,3$; swigen 16,$46 ;$ swert 18,137 ; swigen ib. 280 ; switzen 19,26 ; swigen 24,$102 ;$ swert 25,116 ; swer adj. ${ }^{*} 26,51$; swers v. 27,55 ; swer adj. ib. 56 ; swer s. ib. ${ }^{*} 208 .{ }^{*} 235$; swer v. ib. 111,151 ; swer $\mathrm{s}$. 28, 95. 99; swigen ib. 28; swers ib. 123 ; verswunden ib. ${ }^{176}$; swer adj. ib. *392. 530 ; swebende 30,1 , swers $\mathrm{v}$. ib. 40 ; geswuer 31,82 ; swartzer ib. 94 ; versweren ib. ${ }^{*} 121$; swer $32,{ }^{*} 16$ a.; swebst ib. 32 ; verswigen $\mathrm{ib}$. ${ }_{7} 6$; sweren 33,4 ; swarzwald ib. 11 ; swer v. ib. 107 . $\left.34,34.37,53 .{ }^{1}\right)-$ Kuchimeister: $26 s w$ : gesworn 6, 125. 268. 324; Switz 22; beswert 73; swöster *74. *164. 176; geswigen 101; verswigen *105; geswand 107;

1) Ebenso C sw: swer 38, 131. 142; swers ib. 159. 
swirirent 138. 214 ; swöstren 165; Swarzenbach(3) 193.194. (2) 241. 252; geswiiren 214 ; swior 219.255 ; sweren (verb.) *219 gegın 13 sch: schwü 10, 247; schweren (verb.) 22, 212; schwamm (verb.) 51; schwïrent 122 ; Schwaben 133 , 198 ; verschweren $* 155 ;$ Schwarzenbach 187 ; schwiren 212 ; schwenkel 220 ; schwand 228. - Stretlinger Chronik: fast nur sw: swanz 7, 1; swert 8 , 26. 9, 19. 56, 9. 14. 70, 17; swei/s 9,5 ; swanger 12,5 ; besweren $15,17.58,3.60$, 30 ; beswiir 15,28. 56,16; verswand *16, 23. *33, 5. 43,29. 73, 27. 137,11; beswornen 33,29 ; beswerni/s $35,29.38,10.48,20$. 49,17(2). 116, 7 ; swarzen 37 , 1. 39,21 . 79,22 ; swür 42,8 ; swigen 45,16 ; swuirent $49,22.80,25.88,19$; swester 54, 19; geswal 55, 20; besworn 56, 9. 102, 24. 103, 16. 104, 28. 127, 17. 128,10 ; sweig 69,4 ; sweigte 69,14 ; swar 81,17 ; swetzen 85,8 ; besweren 85,9 ; swig 110,12 ; swarz 112,16 ; swimmen 122,4 ; swiren 132 , 28 ; swende 149,8; swendi 15S,21; swerrent 163, 27 gegen nur 2 schw: verschwand *20, 21; schwimmen 121,20. - Ulrich von Richental: 56 sw: Switz 18; Swilzer 66; swartzen 28. 80. 85. 100. 101; swär 38; sweher 38 ; swinis 40; in der Swartzen hof ${ }^{*} 44$; Swegen 51 ; Sweden 53. 162. 201 ; swiorend 54 ; gesworen 66. 69. 72. 89 ; sweren 68. 70 (2). 105. 106; swior 68; swereni 70. 116; Swartzburg 66; Swartzach 91; Swaben 69. 184. 208; Swauben 79; swert 93. 97. 99. 105 (2). 107. 109. 200; swantz 100 ; swigen 105; Swedur Kobin 184; Swalbach 194; Hanns Swartz 197 (2); Swostery 201; Swidwa 202; sworn 211; Swager 211; Swiger 211. 212; Swelher $213^{1}$ ) gegen 65 șchw: schwior 14. 55(2). 70 (2). 71. *94; geschworen 18. 70. 149; geschwornen 135; geschwir 55; schwưren 53. 66. 71. 106. 107. 110 ; schwiorint 56. 71.102. 110; -d 96. 135; schweren 71 (2). 158; schwert 16. 78, 80. 106. 108. 134. 144. 146. 190; schwär 17. 102; Schwartzach 34. 38; Schwartzen 37. 45. 48. 83.199; Schwartz 48; schwartz 124; Schwartzburg 145; Schwartzenburg 193(2); ain lb. schwinis 40; ain lb. schwini wilprat 40 ; schwecher 40; schwechern 102; schwechrend 141; überschwenklich *42; schwigen 55. 137; schwitzen 131; Schweden 154. 207; Schwaben 159 (2). - Legende vonS. Idda von T'oggenburg: nur schn: Schwaben Al. 12,$173 ;$ schwanz ib. 174 .

B) Merswin: nur sw: swerliche 29.67.132; swere 80.84 .87 (2). 97; besweret 133 ; swigende 32 ; geswinde 41. 53. 69 (3). 71. 80. 81.85 (2). 88 (3). 89 (4). 91. 94. 98. 101; swinde 118 (2); swarme 69 ; überswenkende 113. 124. 127. 128. 129. 132. 135; swarc 130. 131; geswechet 136. Aber daneben schreibt er in nachstehenden fällen $z w$ für $s w$ : zwarc 16. 17. 70; zwarcer 16. 17; zwerc 58 (2). 67; furzwigen 26 ; furzwiget, -ent 58 ; zwigen 64 ; zwerende 52 ; zwach 64 ; zwerliche 68 ; zwere 69 . - Els äs is ch e predigten, Dankrotzheim, Regel des hl. Augustin: nur sw.

r) A ugsburger stadtbuch: nur sw. - Ingold: 23 sw: swert 24 (2). ว1, 66 (3). 71 (3). 78; swerler, swertern 71 ; swaren 34.55 ; swär 73 ; versweren ${ }^{* 53} .{ }^{* 55} ;$ sweren 55 ; rokswantz 67 ; swantz 67 ; swester 68 ;

1) Ich habe hier und in der folge die oben befolgte ordnung der belege aus den denkmälern mit rücksicht auf III insoweit verlassen zu mïssen geglaubt, dass ich mit möglichster berücksichtigung ihrer folge im denkmale die wörter derselben sippe znsammenstellte.

Beitrüge zur geschichte der deutschen sprache. XVII. 
sway/stuch 69; gesweig 81 gegen 40 schw: schwartzer 6; schwartz 9 (2). 68 ; schwerten $i$; schwert 9. 24. 29. 30. 31. 34; schwester 12. 24 ; schware 8 ; schwärer 9 ; schwär 16. *54; schwären 39 . 73; schwärlichen 50 ; schweyget 10. 18; geschveyget 10 ; geschweigend 18 ; verschweigen $*_{5} 9$; geschwigen 81; schwer 14; schwerin 53; schwach *22; schwachen 65 ; schwantz 25. 32. 67 (2). 72 ; schweinmisl 40 ; schwein 41 ; schweinen 49 ; verschwand *52; schwam 69. - Conrad von Weinsberg: nur sw. - 0 tt Ruland: 5 sw: swager 4; swebischem 6; swarz 6; Sweller (n. pr.) 11.16 gegen 6 schw: Schwindenbach 7 (n. l.); schwartz 20. 24. 33; schwecher 'schwager' 35 (2). - Hermann von Sachsenheim, Nörin: 106 sw: swer 24. 848. 1190. *1671. 2198. 2494. 2814. 2947. 3150. 3328. 3910; swer (subst.) 1027; swere 1894. 2716; besweren 1397; beswert 1410. 2465; Swiczer 153. 434. 2490. 27i0; Swicz 497; beswuor 167; swuor*1002. *1539. *1546; sweren 2659. 3556; swieren 2487; gesworn 1612. 1724. 1911. 2011. 2395. 2469. 3632 : swert 236. 332. 459. 1096. 22114. 4116; swerten 1081; allerswerczsten $*_{2} 29$; swarcz *304. 903. 907. 1224. 3415. 3750. 3773. 5116; swarczes 2172; swarczen *5261; swaig 316. 720. 2540. *3918; swig 350. 701. 1576. 1707. 2996. 3579. 3880. 3912. 3956. 5406. 5i72. 6012; swigen 12/9. 1823. 2059. 2363, 2861. 3883; geswig 2051; swigt 2422 ; verswant ${ }^{*} 490 .{ }^{*} 2994$. ${ }^{*} 4033$; Swab 529; Swaub*1771. *1851. 2014. 2017; Swaben pl. 2367. 3489; Swaben land 1661. 1865; Swauben 2344. 3537. 4098; swann' cygnus' *684; swebt 684. 874. 2848; swebet 2854 ; widerswaiff (subst.) *994; swin 1357. 4160 ; geswind 2614. 3798; swilg 2180 (adj.); Swarczwald 3726; swach 3842. 4135 gegen 44 schw: schweren 145. 5268. 5396. 5673. 5715. 5749; schwur (verb.) 5758; geschworn 5696; schwer (adj.) 3812. *5078. 5197. 6065; beschwer 2656; Schwiczer 2485; schwarcz 704 (8c). 5046. 5717; schwarczen 5213. 5616; schwigen *549; schwaig 244; schwaigen 5809; schwig 1011. 1934. 4215. *4349. 4575. 4616. 4662.5780; Schwaben 4748 (land); Schwaben (volk) 4748. *5456; Schwebschn 1850. 3848; schwebscher 5955 ; Schwaubenland 5694; schwan 890. 5618; schwalb 4422; schwach 4987; schwester 1016. 4582; Schwapelrü/s. - Der goldene tempel: nur schw: schwach 466. 831; schwarczer 1258; schweben (inf.) 126; schwebt 274; -et 789. 804; schwebent 23. 515; schwer 593. 641. 758. 838. 1057; schwar 450; schwer (8ubst.) 619 ; schwere 794 ; schwestern 637 ; schwig 798. 863; schwigen 873. 1039; geschwigen 1217; schwingen 54; Schwicz 1284. - Jesus der arzt: nur sw: swer (subst.) 14; swer (adj.) 30 ; swerl 126. - Ka ufringer: 104 sw: swär (subst.) 1, *59. *355. 414. 3, 1. 186. 306. 534. 4, 263. 5, 43. 198. 225. 266. 442. *658. 7, 250. 8, 408. 9, *63. 10, 48. 11, 41. *181. 500. 557. 12, 155. 13, 95. 426. 14, *4. 95. 231.412. 705. 753. 761. 15, 97; swar (adj.) 3,*107. *399. 477. 499. 4, 330. 13, 167. 14, 574. 16, 107. 708. 794; sware 14, 299; beswaren 16, 84; beswer 16, 204 ; beswärt 5,643. 10, 41; swach 3,188 . *656. 8, 395. 11, *230. 14,410. 16, 184 . 383. 434. 656 ; swachen 13,262 ; geswacht 6,16 . 26 ; ungeswacht 5,72 ; unbeswacht 13,128 ; verswach $7,{ }^{*} 72$; swanger $14,87.127$; swank 16 , 592; swebt 3, 700. 4, 34. 7, 382. 8, 24; swaif 7, 226. 15,65; swert 6, 141. 238. 11, 291. 947; swerlzsleg 4,172; swester 14, 361. 421. 426. 432. 470. 17,93; sweigen 7, 243. 17,257 ; swaig 1,119 . 3,199 . 719 . $13, * 187$; verswant $1, * 322$; ver- 
swendet 17,$265 ;$ swang $3,606.13,{ }^{*} 227.14,341.363$; geswizet 9,91 ; swei/spad 9, 89. 119. 189; gcsworn 7, 134. 164. 9,1117. 10,46. 11,417 gegen 15 schw: schwar (subst.) 2, 242. 3, 116. 4, 48. 482; geschwachet 14, 293; schwaif (verb.) 5, ${ }^{*} 116$; schwieff $14,{ }^{*} 596 ;$ schweig $6, *^{*} 249$; verschweigel $17,{ }^{*} 251$; verschwand $1, *_{43}$; verschwinden $10,{ }^{*} 46 ;$ verschwendt $17,{ }^{*} 25$; schwuor 9, 57. 11, 443; schwür 15, 11.

b) Im bairisch-österreichischen sprachgebiet.

1. Urkund liche belege:

§ 22. Friderich der Swantz 1304, Urkb. d. 1. 0. d. E. 4, 454 no. 495; Swager, swester, geswislereiden 1340 ib. 5 no. 88 ; swester 1340 ib. no. 350; Heinreichs des Swarlzen 1346 ib. 6, 534; sweren (adj.) 1354 ib. 7, 358 no. 348; ze Swammern, swager 1356 ib. no. 453 ; sch wester neben swester (3) 1361 ib. 8 no. 14; sweyn 1361 ib. no. 24; Swans, geschworn neben geswornen 1361 ib. no. 30; Seybots des Sweinpekchen 1361 ib. s. 49 ; swager, Swerperg 1362 ib. no. 61 ; beswerung 1362 ib. 8. 107; beswert s. 226 . 228 ; beswernuss s. 226 ; sweren (verb.) 8. 227 , 1365 ib. no. 223 ; schwecher (subst.) 1365 ib. s. 233 no. 229; ungesworn 1366 ib. no. 281; geswistriden 1369 ib. 8. 410 no. 411 ; swein 1369 ib. 8. 437; sweher, swester 1369 ib. no. 450 ; gesworn 1370 ib. s. 455 . - A us weistümern: swerzvehen 'angriff mit dem schwerte' 1342 , bergr. v. Gastein Ö. w. 1, 199; swaidler 1346, Gastein bergr. ib. s. 201; schwein, schwaigen, schwenten aber versweigest 14. jh., Pillersee 'l'. w. 1,90 ff.; swein 1387, Telfes ib. 1, 279; Peter Schwertz 1398, Pfuns ib. 1,294; gesworen 1411, 'Irins 'T. w. 1, 292; swein, Swent, swenten 1434, Brandenberg ib. 1, 135; swimmet, Schwaiger ca. 1440, Wildschönau ib. 1, 133; schwär, swaiger, Swertan 15. jh., ib. 1, 88; schwaig (2), beschwart ca. 1440, Stumm ib. 1, 139; swar unbeswärt, swaiger, dienstswein 1462, Axams ib. 1, 253; ver schwige 15. jh., Abram ib. 1, 201; swein (0.), sweinanger 15. jh., Innsbruck ib. 1, 231; Swartzenthals, schweinbern ib. s. 25, 15. jh., Flaurling 'I. w. 2, 24; beswäret (4), sweren, swein 15. jh., Zillerthal Ö. w. 1,317; swarzwild, swein (3), swarlich (3), beswärt, beswärung, swaigern, swaig 1497 u. 98, Mittersill ib. $1,283 \mathrm{ff}$.

2. Belege aus literarischen denkmälern:

$\S 23$. 0 swald von Wolkenstein: 168 sw: swerer $1,1,10$; swer (adj.) ib. 1,24; swam ib. 2, 28; swais ib. ${ }^{*} 5,4$; sbere ib. 6, 5; swerl 2, 2, 32 ; swymmen ib. $3,1.3,14$; Sweden $3,1,9$; sbartz ib. 2, 17; swer ib. 3, 7; geswecht ib. 3,$19 ;$ swach $4,3,10 ;$ swein ib. 3,3 ; swartze ib. 3,18 ; sweren ib. 3,20 ; swacher, swancz ib. ${ }^{*} 4,2 ;$ Swaben ib. 4,4 ; swert $6,1,111$; swantzen ib. 1,167; swer (adj.) 7, 2, 14. 8,2,10; gesworen 10, 5, 2; swer (adj.) 11, 3, 24 ; sweren 12, 3,34; Swab 13, ${ }^{* 7,1}$; swcre ib. 4, 2 ; swaig ib. 4,3 ; versbell ib. $* 7,9$; switzen ib. 9,2 ; geswaigen ib. 10,2 ; swach $15,3,9$; versweigen $16, * 1,5$; gesbechet ib. 1,8 ; versbunden ib. ${ }^{*} 1,16$; Swongau ib. 4, 8; swach $17,3,7$; versbigen ib. $3,{ }^{*} 37$; sbartz ib. 4,27 ; sbern ib. 5,7 ; sbarlz ib. 5 , 18 ; sbeimen $18,4,8$; sbaigen $19,2,13$; swer $20,1,18$; swert $21,3,1$; swig $23,1,1$; sweren ib. 3,6 ; swachen ib. ${ }^{5}, 4$; sbachlich $24,2,6$; sberlich ib. 3,6 ; swach $25,{ }^{*} 2,3$; beswert ib. 5,11 ; swere 26,44 ; swachen ib. 48 ; swer (subst.) ib. *96. ${ }^{*} 165 .{ }^{*} 315$; swacher ib. 103 ; swerer ib. 115 ; swert ib. 
147; swachen $29, *_{1}, 39$; verswigen ib. $2,{ }^{*} 29$; ungeshechet ib. 24 ; sbartzer $30,2,28$; versbunden ib. $2,{ }^{*} 39$; swank ib. 3,17 ; swere $31,1,21$; erswetzen ib. 4, *31; verswunden $33,1, * 7$; sweren $34,3,11$; swartzer $35,2,36$; sbammen ib. 3,1 ; peswärt $36,6,4$; sweig $39,2,20$; swachen $45,1,3$; geswaigt 47,1 , 2 ; swer 48,3,3; verswigen $51,1,{ }^{*} 19$; swer (subst.) ib. 3,1 ; geswulst 52 , 2,6 ; swert 54,1,3; geswind 56,1,14; swindt $61,1,7$; sweig ib. 1,9;62,1, 1 ; swer (subst.) $65,2,10 ; 66,3,15$; swer $68,2,9$; sweig $69,1,1$; swartzer $72,1,2$; swartzen ib. 1,8 ; swer (subst.) ib. 2,13 ; swestern $75,1,3.12$; swebt 76, 2,15; sbammen, swemmelein 77, 2,11.12; swester ib. 3,14 ; sweres (adj.) 77,4,15; ungeswacht $83,1.12$; sweren (adj.) $86,1,6$; swach ib. 6, $*_{19}$; swerlicher 87,2,21; swer (subst.) 88, 2, 2; Swaben 89,1,8; Swebin ib. 21 ; versbunden $90,2,{ }^{*}$; Swaben $91,2,14 ;$ geswier ib. 3,12 ; verswunden 94, 2, "2; Sweden 95, 1, 3 ; swantzt ib. 1,16; swār (adj.) ib. 4,8; beswäre ib. 5,10 ; swebt 100,1, 1; swach ib. 2, 3; swer (subst.) 101, 1, 16. 102,1,8; gesnymmen 103,1,13; swer (verb.) 105, 1, 10; beswer ib. 3,10 ; swert 106, $3, * 16$; swer (subst.) ib. 4,12; swertlen ib. 5, 9; swachen $106,10,10$; swanck ib. 10,12 ; swerlich ib. 10,12; geswindlich $107,3,9$; swerlich ib. 4,5 ; sberlich ib. 4,12; sbais ib. 5, 6; swanger 108, 1, 3; swerlich ib. 3,8; swer (adj.) ib. 5,18 ; swindt ib. 7, 1; swer (adj.) ib. 7, 13. 14; swer (adj.) 109, 1, 17. ib. 3,15 ; swecht ib. 4,16 ; geswechet $110,2,10$; swantz ib. $3,{ }^{*} 16$; swennt $111,3,17$; swirt $112,1,{ }^{*} 13$; swecht ib. 1,15 ; swarlz ib. 2,3 ; swach ib. 2 , 18 ; swucher 114,2,7; swer (adj.) ib. 3,3 ; geswilt ib.; umbeswaif ib. 3,7 ; sweren (adj.) 115, 4, 7; swer (adj.) 116,1, 2; swer (subst.) ib. 2, 7; swachen ib. 5,2 ; swartz $117,2,7$; swester ib. $4,{ }^{*} 12$; swachem ib. 5,8 ; sweigen ib. 7,11; swach 118,1,3; swachs ib. 1, 17; swester ib. 7,*17; swerleich 119. 2,10 ; swachlichem ib. 2,18 ; sweren (adj.) ib. 3,10 ; swerlich 121,50 ; swärlich $\left.{ }^{1}\right)$ 122, 2, 5 gegen 4 schw: schwänlz $6,1,{ }^{*} 49$; schwacher $25,4,2$; geschwacht $91,2,4$; erschwillt 114, 3, "5. - Schiltberger: 14 sw: swager 9,9 ; swach 18,9. 17; sweher 18,29. 34 ; swäre 37,15 ; swert 37,16 . 90, 17; geswellen 50,2 ; swartzen $54,30.63,7$; swarlz 69,29 ; swangern 89,26 ; palswam 102, 23 gegen 50 schw: schwur 5, 20; schwuren 13,8; geschworen 27,5. 111, 2; schweren 61,36 . 93,13; schwerdt 11,16; schwert 21,1. 37, 14. 17. 43, 11. *15. 61, 35. 85, 15. 86,12. 90, 19. 98, 10.11. 18.101, 21; schwertt 21, 2. 23, 6, 28, 7; schwester 12, 8. 15; schwartzen 43, 27. 44, 9. 46, 9. 54, 26. 55, 5. 63, 1. 22. 26. 84,18 . 85, 22. 91, 3. 128, 32 ; schwarlz 57, 10. 69, 30. 80, *11. 84, 33. 85, 10; schwerlz ${ }^{*} 80,17$; schweifs 71,12 ; beschwercn 8 ;, 7 ; beschwert 89,3 ; schwanger 91,29 ; schwcinefleysch 92,9 ; schwein 100, 33 (2). - Beschreibung einer seereise: nur sw: gesworner 16. 225; swipogen 269. - Niirnberger polizeiordnungen (13. u. 14. jh.): nur sw. - Tucher: erste hand 77 sw: gesworen (rr) 71, 12. 79,4. 11. 17. 90, 19. 131, 38. 147, 27. 277, 26. 281,3 ; sweren 90,12 . 23. 216,34 . $272, * 21$. 274,2 . 279, *13. 286, 26 ; svere $217,{ }^{*} 5$; swell $(l l) 198,{ }^{*} 22$. $234,{ }^{*} 25$; geswell 74, 13. 283, 24; geswelle 258,12 ; swelle $222,{ }^{*} 13$; swellen $229,{ }^{*} 28$; aufswell 231,11 ; swer (adj.) 116, 21; beswert 199,6. 201, 32. 272, 15; swarlze 68,1; Swarlzen 68, *36. 296, 34; Swarlzverber 159, 36; Swarlz 152,5; Swartz-

1) Nur in W. 
heinz 15:,37; richtswert 119, 20; swarten $77,21.31 .32 .121,17$; swipogen 157, 22. 28. 166, 23. 167, 1. *4. 5. 7. 19. 179, 14. 21. 22. 25. 32; sweher 264, 14. 265,18 ; swein 267,34 ; sweinen $270,{ }^{*} 27$; sweinensmaltz 249,12 ; sweinstall 267, 33; Sweinmarckt 113,30. 143, 22. 200, 29. 203, 16. 251,3,14. 26 , 25. 290, 23. 33. 291, 5; Sweinaw 206, 11. 208, 22 . 210,4; Swoben 141, 33. 145,18; Swan 155, 32; Sweicker 157, 14; Swygershoff 210, 2 gegen 14 schw: schweren 70,13. 272,15; schweigen 67, 23; schwig ib.; slillschweig ib:; schweissen 100,19; geschwungen 109,16. 19; Schwingsherlein 269,10; schwipogen 128,17. 150. 36. 178,35; Schweinmarckt 76,13; Schweinaw 206,11. Zweite hand nur sw: sweren 312, 7. 17; gesworen, gesworn 311 , 19. 320,13 . 328,36 . 332,2 ; sweher 310,23 ; swynbogen 331,12 . 13. 18. 333, 4 ; Swingsherlein 314,7 ; swachen 313,29 ; Swab 333, 28; swervischen 324, 5 ; Swobach 316, 1.

II. Im mitteldeutschen sprachgebiet.

1. Urkundliche belege:

§ 24. gesworn 1329, Meissner urk. no. 34 ; gesworyn 1352, ib. no. 41; besweren 1357, St. Afra no. 210; 1367, Meissn. urk. no. 50; gesworn 1380 , ib. no. 55 ; geschwornen 1386 , ib. no. 57 ; gesworn 1387, ib. no. 59; swestir 1391 ib. no. 61: gesworen, sweren 1403, ib. no. 67; Swemicz, besweren 1427, St. Afra no. 264; gesworne 1433, Meissn. urk. no. 79; 1441, ib. no. 92 ; sweren (adj.) 1447 , ib. no. 102 ; gesworne 1456 , ib. no. 113 ; gesworn 1457, ib. no. 115 ; sweren (adj.) 1474, St. Afra no. 296; schbert gr. $\left.{ }^{1}\right)$ schlossbaurechnung von 1477, Meissn. urk. s. 95; Swertfeger 1480, ib. no. 138. $142 ; 1455$, ib. no. 146 ; swestern 1506, St. Afra no. 328 ; Sch wedler, Schwertfeger 1508, ib. no. 329.

2. Belege aus literarischen denkmälern:

\$25. Johannes von Frankenstein, das Buch vouguter speise, Offenbarung Johannis, Ritterspiegel, Stolles 'Thür.Eifurt. chronik: nur sw. - Miscellanhs. (Pietsch-Rïckert, Entwurf 144) hat in folgenden fällen schw: schwankil, beschweren 1, 107. 108; schwer 1,105; verschwenden 1,132.

\section{B. sp, st, (sk).}

$\S 26$. Hier handelt es sich um diejenigen verbindungen eines $s$ mit einem consonanten, bei denen die neuhochdeutsche schreibweise die $\check{s}$-aussprache unbezeichnet lässt und die im gegensatze $\mathrm{zu}$ den unter A behandelten nicht nur anlautend sondern auch inlautend auftreten. ${ }^{2}$ )

1) Münze.

2) Hier werden nur belege der schreibung mit sch vorgeführt; es möge sich dieses verfahren rechtfertigen in hinblick auf das starre festhalten der $s p$, st in der heutigen orthographie im gegensatze zu der nhd. schreibung von schl, schm, schn, schw. 


\section{I. schp.}

\$27. Anlautend: Die Stäfner öfnung (15. jh., Grimm, Weist. 1, $45 \mathrm{ff}$.) bietet 5 schprechen gegeu 7 sprechen, fürschprechen gegen unuersprochen. - 0 t t R u land: cz' Schpir 'zu Speier' s. 2. - In lau tend: Ehingen: 1schpanien 12,14. 16, 23. 17,3. 7. 21. 18,19. 20,16. 26,1.8. 18. 27, 28. 28, 16. 20; Ischpanisch 27,34 .

\section{II. scht.}

§ 28. Anlautend '): Fab. Frangk s. oben s. 225. - Inlautend: Urkunden von Basel: ernschlich 8. 530, 4 ; geischlich 532 a. 1393 no. 489; exemplar A. - St. Galle n: gaischlich 1340 no. 1386.1344 no. 1421. - Schwäbische urkunden: gaischelich 1350, Mone 10, 254. 1401, ib. 11, 217. 1411, ib. 105; ernschlich 1447, ib. 110. Hierher vielleicht das engesclicher (K. Hofmanns text: engestlicher) der handschrift von $\mathrm{L} u \mathrm{t}$ w in $\mathrm{s}$ Adam und Eva v. 2532. ${ }^{2}$ ) - Wirklich scht geschrieben finde ich bei: 0 tt Ruland: in der faschten mes s. 1 neben fasten ib.; umb ach mischlin bater noschter 'rosenkranz aus mistelholz'; herschtmess s. 2 (die folgenden einschreibungen weisen durchaus mistlin pater noster und herstmezz auf.) - Ingold: fierley mischt, das was schaufmist, leomist, affenmist und schweinmist 40 ; verluscht 50 . - Wackernagel: hieschischt 142 (Weinhold s. 15). - Wit te $\mathrm{n}$ weiler, Ring: $O$ we, min hord totscht du mich 12c, 46. - Richental: grauf von Diescht $\mathrm{K}$ (Tiest $\mathrm{A}$ ) 194. - Konrad von Megenberg, Buch der natur: der nebel isl aller schade scht A (14.jh.) 8. 96, 12; gesündis cht A 104, 5; zeletscht 161, 12 A; let scht A 185, 10; vor froscht A 224,16; geröscht B (1377) 350,18. 351, 11; röscht A 350, 24. 381, 12. - 0 s wald v. Wolkenstein: 4, 3, 3 faiste swein, gemescht von Kyb W, gemescht von Kleyb $\mathrm{X}$.

\section{III. sk.}

§ 29. Hier können naturlich fast nur fremdwörter in betracht kommen, da sk schon lange zu sch (r) geworden war, wenn auch die schreibung $s c$ noch in unserer zeit fortlebt.

a) als schk: Register: Muschkatblüt 18, 7 (Mus stkatblüt 79). U r.k. จ. Oberöst. 8 s. 393, a. 1368: hundert phunt und dreyschk phunt

1) Neben scht findet sich (vgl. 8. 225 anm. 2) sth geschrieben; wohl in ähnlicher weise zu beurteilen wie die schreibung in unreth (unrecht), die schon in spätahd. denkmälern sich findet (vgt. Weinhold, Mhd. gr. $\S 202$ ). Demgemäss wird auch bisthum (bistum) Stolle s. 91. 132.146 zu beurteilen sein.

2) Beizuziehen wäre auch Basler urk. s. 71,7 ff. anno 1422, 0etlingen: als denn gefragt ist von der vischentzen wegen, spricht er, daz er in dem obgenanten zyte einem knechte.. lih die vischentzen...., also daz er davon alle wichen einen diensch visch geben sölte. Vgl. auch dynschedagh Lac. 3, 965 (Weinhold, Mhd. gr. ${ }^{2} \S 210$ ). 
wienner phenning (2). - 0 swalt $\nabla$. Wolkenstein: 95, 4, 2: tomaschk (W X ohne k). - Suchenwirt: Gaschkonien 18, 143. - Buch von guter spei se: muschat 27 a. - Stolle 63, 10: sundern er wolde selbest korfurste, konnig, keiser und bobist sin, vnnd alle korfursten vnd ouch andere fursten worden sine knechte vnd schtileuen.

b) als schg: Ehingen: Damaschgo 13, 24. 26. 30. - Katzenelle n bogen: (wir) qwamen in porte Fischgardo ${ }^{1}$ ) 369, aber Fischardo 369, 5. - Rich ental: der cardinal von Flischgo 127. 128; her Woschga von holabrat 152; dominus Blasius lusinensis episcopus in Tuschgania 161; Tuschgan 201, doch Tuschan 50; Mollischgo, Sawoschgi, Prognewischgi 202; Karwischgi, Kalischgi 186 (pöln. namen).

$\S 30$. Im allgemeinen können wir aus dieser zusammenstellung vor allem nur das bestätigen, was die grammatiken (oben s. 225) lehren, nämlich die zunahme der schreibung mit sch gegen das 15. jh. hin. Es wird aber auch aus der ubersicht deutlich, dass diese schreibung um 1300 begonnen hat (s. oben § 2. 4. 6. 8. 10.12.14. 16. 18. 20. 22). Wir sind jedoch im stande, durch vergleichung der entwicklung dieser schreibung in den einzelnen dialekten und bei den einzelnen verbindungen eines $s$ mit einem consonanten untereinander dieses resultat nach beiden seiten hin zu ergänzen.

§ 31. Es lehrt nämlich ein vergleich in dialektischer beziehung, dass im md. und els. ${ }^{2}$ ) die alte schreibung mit $s$ viel beliebter ist als in den andern dialekten (vgl. § 9, $\beta .15 \beta .21 \beta$.)

$\S 32$. Vergleicht man die consonantenverbindungen untereinander, so kann man mit bezug auf ihre stellung im worte (anlautend oder inlautend) drei gruppen unterscheiden, bei denen die schreibung mit sch stattfindet:

a) Nur im anlaute finden wir schl, schm, schn und schw; es liegt in der natur der geringen zahl und des seltenen gebrauches der wörter, die diese verbindungen auch inlautend aufweisend, dass sich keine belege mit inlautendem $s c h l, s c h m$,

1) Porto Viscardo an der nordspitze von Kephalonia.

2) In bezug auf die schreibung des $s c h l$ (vgl. oben s. 228) weicht dieses allerdings vom gebrauch des mitteldeutschen ab.

s) Eine superiorität des alem. mit ausnahme des els. hinsichtlich der schreibung mit sch vor dem bairischen kann man vielleicht auch vermuten (vgl. sn s. 235 f.), der unterschied ist aber nicht so klar wie der oben erwähnte. 
schn und schw bieten. $\left.{ }^{1}\right)$ Doch uberwiegt die schreibung von $s w$ statt des modernen schw auffallend gegenuber den im verhältnis weit häufiger geschriebcncn schl, schm, schn, wobei ganz abgesehen wird von den $s w$ der verallgemeinernden pronomina und pronominaladverbia, die mit sehr geringer ausnahme (schwaz Augsb. stb. 24; schwelich Wack. pr. 34,21; schwer Zur. jahrb. 47, 12 ; schwo M. S. A. 267, 12 ; vgl. Weinhold AG. § 190) durchaus $s w$ zeigen. ${ }^{2}$ ) Es ist selbstrerständlich, dass entsprechend den heutigen verhältnissen - die heutige orthographie kennt ja kein schp, scht, (schk) - die schl, schm, schn und schw gegentiber den andern verbindungen weitaus uberwiegen.

b) Nur im inlaute findet sich scht, wenn wir von den ganz späten belegen für den anlaut absehen.

c) Sowohl im an-als auch im inlaute finden sich schp, schk.

$\S 33$. Es ist wohl selbstrerständlich, dass die veränderung der schreibweise auch eine veränderung des lautwertes voraussetzt, dass also in unserem falle in der zeit, wo die ersten belege mit schl etc. auftauchen - und wenn wir der orthographie eine gewisse trägheit im nachfolgen der sprachlichen entwicklung zuerkennen, schon fruher - die aussprache $\breve{s}$ conform der heutigen in geltung kam. Wenn eine änderung in der schreibweise bei $s l$ schon im ahd. (vgl. Braune $\S 169$ anm. 3) insofern eintrat, dass wir hier zwischen dem $s$ und $l$ ein $c$ oder $k$ eingeschoben finden (3 sclâf, sclahan, sclahttu, kasclactôt, sclectêr, sclehtêm Hymnen; skluog Ludwigslied 52; piscluoc Eirstes Reichenauer glossar; sclâphun Mainzer beichte; sclahda Physiologus), so wird dies mit Scherer, zGDS. 127, dem auch Braune, Abd.

1) Es kommen fast nur wörter mit -sl-, -sn- aus -sel-, -sen- in betracht: so auch -sm- fismen 'sich unruhig bin und her bewegen' Schw. id. 1, 1081; flismen 'flikstern' ib. 1212; frismen, chrismen 'erbeben' ib. 1329 ; lismen 'stricken' Schm. Wb. 1,1513 (gli/smet Voc. Archon. f. 20; glismet Cgm. $690 \mathrm{f} .158^{\text {b. }}$ Cgm. $826 \mathrm{f}$. 159) und -sn- in fisner 'hirtenknabe für schafe und ziegen' Schw. id. 1,1081; lusnen, lisnen 'horchen' Schm. 1, 1515. Lexer 1, 1991; überall weist das heutige $s$, nicht $\check{s}$, dieser wörter darauf hin, dass einst ein vocal hinter dem $s$ stand.

2) Ausgeschlossen wurden hierbei noch die $s w$, die in der composition entstehen, wie bei den ortsnamen auf -wil, -wiler, -wang, wenn ihnen ein genitiv auf $s$ vorausgeht (vgl. unten s. 266, anm.1). 
gr. $\S 169$ anm. 3 folgt, wohl noch immer am besten dadurch erklärt, dass das $l$ durch assimilation an das $s$ stimmlos wurde, wobei das explosivgeräusch des $l$ im $k$ seinen ausdruck fand. Dieses $s c l(s k l)$ darf also nicht, wie es Weinhold, Mhd. gr. 2 $\S \S$ 206. 208. AG. $§ 190$ und neuerdings Leitzmann, Beitr. 14, 514 ${ }^{1}$ ) tun, mit unserem schl in zusammenhang gebracht werden. Ucber einen analogen einschub eines $k$ zwischen $s$ und $l$ im slavischen vgl. Miklosich, Gramm. ${ }^{2}$ 283. Beiläufig sei auf die scl, scm, scn des ags. hingewiesen (Sievers ${ }^{2} \S 210,1$ ).

$\S 34$. Wie misslich aber immerhin die zeitbestimmung einer lautlichen veränderung nach dem kriterium der schreibung ist, lehren uns ja deutlich die verhältnisse wie sie z. b. beim sc vorliegen. Hier sind schon aus sehr früher zeit, aus dem 8. und 9. jh., Braune $\S 146$ anm. 2, sch nachgewiesen. Es ist möglich, dass wir hierin eine vorstufe des mhd. $s$ haben, sch aufgefasst als $s+c h=$ nhd. $c h$ in $i c h$, aber dann ist die bestimmung der zeit, in die wir den übergang des $s+c h(=i c h$ laut), der vorstufe, in $\breve{s}$ setzen wollen, eine sehr vage. Doch es wäre auch möglich, dass wir in diesen sch nur eine orthographische variante zu sehen haben, sowie wir (auch in den denkmälern die sch bieten) im ahd. in fränk. quellen neben $c$ auch $c h$ und in obd. $c h$ neben $c$ finden (Braune $\$ 143$ anm.

1) In dem uns hier interessierenden passus des Leitzmannischen aufsatzes: ' $s$ vor $l, r$ und $w$ hat schon den lautwert $\check{s}$ angenommen, wie die massenhaften (!) $s c$ beweisen (Weinhold $\S 190$ )' $-s c$ habe ich die ersten 50 seiten der predigten des 1 . und 2. bd. darauf prüfend nur vor $l$ gefunden - ist vor allem zu berichtigen, dass das deutsche (sowie uberhaupt das germanische, vgl. Brugmann, Grundriss \$ 578. 580) die lautverbindung $s r$ nicht kennt (wohl entstehen $s r$ in der composition von auf $s$ auslautenden stämmen mit suffixen, die mit $r$ beginnen oder mit solchen, von denen nach ausfall des vocals nur $r(r)$ übrig bleibt. Ueber diese verbindung und deren weiteres schicksal im ags. und nord. $\nabla g$ l. Sievers ${ }^{2} \S 180$. Noreen $\left.\S 200,2\right)$ und dass, wenn die lautgruppe scr gemeint sein sollte, diese schreibung $(s c)$ schon im ahd. (Braune § $146 \mathrm{anm}$. 1) 'besonders beliebt' war. Ferner ist darauf hinzuweisen, dass die $s c n$, schn bei Weinhold AG. $\S 193$ (es sind dies: scwen Griesh. pr. 1, 85; scrvierunt $\mathrm{ib}$; schwene 1,168 ) gegen Weinhold gewiss als $z w$ aufzufassen sind. Es liegt ein rein orthographischer wechsel des $c$ und $z$ vor; man vgl. das umgekehrte in der Basler urk., B. u. 8. 1331 no. $292:$ guszhaden, shilling; ib. 1296 s. 134 twiszent 'inter'; zismatici Richental 158, 4 (vgl. auch Weiuhold, Mhd. gr. ${ }^{2} \S 203$. 205). 
2,3). Am wahrscheinlichsten scheint mir dies z. b. beim Freisinger Otfrid (Braune § 144 anm. 2), der sehr häufig ch für ein $k$ der vorlage einsetzt. ${ }^{1}$ ) Wir sind nun freilich wenigstens bei schl und scht durch zuziehung anderer kriterien in der lage, für das sch den lautwert $\check{s}$ zu erweisen und dadurch auch manches fur die datierung des lautwandels zu gewinnen.

$\S 35$. Beweisend fur $s c h l$ sind die schreibungen mit $s l$ anstatt $s c h l$, wo es auf scl zurlickgeht (Weinhold, Mhd. gr. ${ }^{2} \S 206$. 210. AG. § 190. BG. § 154 ; Birlinger 135.) ${ }^{2}$ ) Den an diesen orten gegebenen belegen seien noch hinzugefügt: Meinauer naturlehre ${ }^{3}$ ): menslich 17, 13، 35; Stretlinger chron ${ }^{4}$ ): mönslich 4,20. 45, 22; Merswin ${ }^{5}$ ): menslich 25, 27 etc. sehr bäufig.

$\S 36$. Beweisend für scht sind einmal analog dem obigen die schreibung a) st statt scht $<s c t$ und zum andernmal die reime von wörtern b) mit scht $<s t$ auf solche mit scht $<s c t$ (zu vergleichen sind die schon oben angezogenen stellen aus (Braune, Weinhold und Birlinger). Dazu fuge ich ad a): Ingold: mist (fur mischt) 37, gemist 52 (aber gemischt 40). Herm. v. Sachsenheim, Mörin: wunst ich 502, wunsten 3353; wust 2077 'wischen'.6) - Hätzlerin: wunst 11, 284. 2, 2580. 5, 22 ; erwünst 2, 7.16, ad b) 7) Herm. v. Sachsenheim, Mörin: mit grossen knütteln als die füst. Ich mein sie haben ro/s ge-

1) Interessant in dieser beziehung ist ein schreibervers in Philipps Marienleben, Wiener hs. 2709, Hoffmann 1211a: 'mercedem po scho laboris' (14. jh.)

2) Doch schon im althochdeutschen zeigt sich $s l$ für $s c h l<s c l$ wie auch st für scht<sct; vgl. Braune, Ahd. gr. \$146 anm. 5.

3) Hat sonst durchaus $s l$, s. oben s. 227.

) Hat bis auf ein schl durchsus $s l$, s. oben s. 228 .

5) Hat bis auf ein sl durchaus $s c h l$, 8. oben s. 228. -- schl entstehen bei ihm in den wörtern geischliche 16,19 n. $\ddot{\text { or }}$. (daneben etwas häufiger geislich) und in erneschliche 47 (aber erneste) als stellvertreter von schtl. S. oben $\S 28$. Ueber sonstige orthographische varianten dieses denkmals in beziehung auf $s$ resp. sch 8. s. 241 .

$\left.{ }^{6}\right)$ der hiibste, pulcherrimus, v. 2223 ist wohl ein schreibfehler.

7) Unsicher, ob hieher zu ziehen, weil neben formen mit sch auch solche mit $s, s s, z$ vorhanden sind: 0 . v. Wolkenst: kreisten : reisten 14,5 . 16. 18, gelust : vertust $119,2.18$. - Hätzlerin: lust : vertust 27 , 350 , verlust : vertust 41,16 . Ich glaube nicht, dass man freisten : leisten W ernh. 205, 19 bei Weinhold, B. gr. $\S 154$ hierher ziehen soll. 
tüscht v. $3574 \mathrm{ff}$. - Hätzlerin: 28,19 glast : vast : erwascht. Demnach ist dem $s$ in der verbindung $s l$ (wegen fleislich Martina, menslich Mein. nat., Mersw.) wohl schon fur den beginn des 14. jhs. der lautwert $\breve{s}$ zuzusprechen; damit stimmen auch die ersten belege für schl (Alem. 1302, Bair. 1312). Die alem. aussprache der inlautenden $s t$ als $s t$ muss wegen Erec v. 1790: lasle : glaste; Barl. 323, 25: erlaste : glaste; Lanz. 2208: wuste : geluste, ib. 3152 wunsten : kunsten spätestens am ende des 12. jh. in iibung gekommen sein.

§ 37. Nach analogie des schl werden wir für schm, schn, schp, schw im anlaute, da ihre ersten belege (vgl. noch ausser oben $\S \S 8.10 .12 .14$. 16. 18. 20. 22. 24 schmalz 1287 H. U. 3,1550; geschprochen 1317, Schreiber, Urkb. d. st. Freiburg und Weinhold, Mbd. gr. ${ }^{2} \S 208$. AG. 190. BG. 154) auch auf das ende

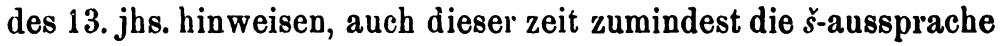
zuerkennen mitssen.

§ 38. Für anlautendes scht war es nicht möglich, einen beleg aus dieser zeit zu bieten; aber die analoge aussprache dieser consouantenverbindung in Ober- und Mitteldeutschland mit der der obigen verbindungen machen diese zeit auch für diesen lautwandel wahrscheinlich.

Der grund, weshalb wir trotz der analogen aussprache doch $s p$, st geschrieben finden, mag wohl darin liegen, dass in den gegenden, in welchen diesen consonantenverbindungen derselbe lautwert sowohl im an- als auch im inlaute zukam, wegen dieser gleichheit kein grund vorlag, die schreibung zu ändern, und dass dort, wo eine verschiedene aussprache im an- und inlaute herschte, die inlautenden $s p$, st hemmend auf die entwicklung der schreibung von schp, scht im anlaute wirkten. Diese annahme der bemmung scheint in dem umstande ihre bestätigung zu finden, dass sich wobl anlautende schp, bei denen im vergleich zu den verhältnissen, wie sie bei st vorliegen, nur sehr wenige $s p$ des inlautes dem $s p$ des anlautes gegenliberstehen, aber keine scht anlautend finden.

$\S 39$. Bezuglich der auffallenden tatsache, dass $s w$ sichtlich therall beliebter ist als $s c h w$, sei auf 8. 264 anm. verwiesen.1)

1) Ueber $s l$ etc. im mittelniederdeutschen $\nabla g l$. Lübben s. 49 , § 38 . 
II.

$\S 40$. Ferner ist $s$ in der stellung nach $r$ zu š geworden. In der schriftsprache sind es nachfolgende wörter, die uns diesen wandel zeigen: arsch mhd. ars, barsch mhd. bars, birschen mhd. birsen, afrz. berser, bursche, mhd. barse f. 'börse', dorsche $\left.{ }^{1}\right)$ f., mhd. torse 'kohlstrunk', herschen mbd. hersen, hirsch mhd. hirz ${ }^{2}$ ), kirsche mhd. kirse, knirschen mhd. knirsen, kürschner mhd. kürsencere, morsch zu einer verbalwurzel murs, Kluge, Etym. wb. ${ }^{4} 237^{\mathrm{b}}$. $238^{\mathrm{a}}$, (wirsch aus mhd. wirs comp.?) ${ }^{3}$ ), wirsching neben wirsing $\mathrm{m}$. (zu grunde liegt lombard. versa 'kohl, wirsing', Kluge $358^{\mathrm{b}}$ ). Oefter finden wir diese assimilationserscheinung in der umgangssprache und in der mundart (s. unten s. 262 anm. 2). Conform der zusammenstellung in I gebe ich hier eine zusammenstellung eventuell in betracht kommender wörter und wortverbindungen zur illustrierung der entwicklung der schreibung rsch.

I. Im alemannischen.

1. Urkundliche belege:

$\$$ 41. Glarus: anderswa 1386 no. 104.1406 no. 137; Rapreswile 1402 no. 132; Rapperschwiler (2) neben Rapperswil (2), Rapperswylen 1429 no. 185; Raperswil 1437 no. 207; Raper schwil 1438 no. 218; Rapreschwil 1442 no. 236 ; Gander schwil 1440 no. 227. - Basel: anderswa 1279 no. 139.1299 no. 196.1339 no. 309.1359 no. 377 (i). 1395 no. 496. 1416 no. 592. 595. 1431 no. 661 ; ander swannen 8. $595 \mathrm{z} .4$; anderswannen ib. z. 10 a. 1400 no. 526 ; Birse (flïsschen) 129 no. 183.1302 no. 20 j. 1323 no. 267.1328 no. 286.1355 no. 359.1392 no. 486 ; uff die Birs matte 1415 no. 588; Birs 1432 no. 669. 1435 no. 674; Birsze 1355 no. 359; Birsz 1424 no. 645 (3). 1435 no. 674 ; Birsiche 1335 no. 298.1343 no. 323.1371 no. 416 ; Birsbrugge 1348 no. 339 ; Birsegg 1373 no. 420 (s. o.); -egke 1404 no. 543; kirsegarten 1299 no. 196; kirszbom 1450 no. 749; Ursule, Ursel (5), Ursulen (2) 1371 no. 415 ; kirsener 1390 no. 474.1392 no. 482.483 .1396 no. 497; Mörsperg 1411 no. 572.1416 no. 592; die mit -wiler zusammen-

1) Natürlich gehört dorsch m. (nach nd. dorsch entsprechend anord. porskr, engl. torsk aus dän. torsk) nicht hierher; doch sei der merkwürdigkeit halber des Smaragdus (abt um 800) dursus erwähnt. Es heisst bei ihm, Du Cange 2,966c: 'Piscis species sunt ho, esox, Dursus, alausa, tracta, lampreda et reliqui'.

$\left.{ }^{2}\right)$ Mhd. $z$ ist schon im 13. jh. mit $s$ zusammengefallen (vgl. Paul, Mhd. gr. $\left.{ }^{2} \S 29\right)$. - Die dem mhd. hirz entsprechende form lebt noch mundartlich (alem. und md.) fort: Regel 73. Heusler, Conson. d. ma. v. Baselst. s. 6.

3) Wenn nicht aus (un)wirdesch entstanden, vgl. Kluge EW. ${ }^{4} 365$. 
gesetzten ortsnamen des typus $-r s$ wiler stets mit $r s 1356$ no. 360.1382 no. 449. 1396 no. $\left.497 .^{1}\right)$ - rsch nur in herschet 1444 no. 720 s. 850 z. 13 ; Birsch 1500 no. 974 s. 1107,15 . - Königshofen: bursierer (amt eines klosterbruders) 1422, Mone 10,121. - Augsburg: rs und rss: in hirs cervus im n. pr. Stolzehirs, das in den urkunden sehr häufig vorkommt (vgl. das register im urkb. v. Augshurg). - Aus weistiimern: anderschwa mitte des 14. jh. Ermatingen ('I'hurgau) Grimm Weist. 1, 239²); 15. jh. Roggwil ib. 177; ander schwo s. 13 neben anderswo s. 14 ib. 4.

2. Belege aus literarischen denkmälern:

$\S 42$. Meinauer naturlehre: herschen: do si (die Römer) vor gewaltic warent, daz si herschetent.. uber alle riche... s. 12 z. 12. Hugo v. Montfort: birssen 9,22 ; wirser 17, 2. 17, 183. - Kuchiweister: Merspurg 16; Raperswile 28; wirst (superlat.) 27; getorst 36; fürsten, anderswa 164. - Neujahrsspiel (Monc Schausp. 2, 378): rsch in anderschwa v. 420 gegen $r s$ in arsloch 135; ein gantzi bursami 170; wirs 448; persönlich 584. - Heinrich Wittenweilers Ring: rsch in herschen: emphfilhst du ir das haus mit sampt, so wyl sey herschen in dem smpt $18^{\mathrm{b}}, 12$. Sogar das genitiv-s nach $r$ als sch geschrieben: ist sey arm, nu hin, daz sey! Dannocht lebt sey noch dabey, sey schlaft dest bas und furcht ir nit vor teupen und vor feursch geschicht 22, 37; $r s$ in ärs (podex) $3^{\mathrm{d}}, 34.4^{\mathrm{b}}, 9$ u. ö.; phersisch 27 $\mathrm{b}, 27.37 \mathrm{~d}, 27.37 \mathrm{~d}, 3 \mathrm{j}$; wirser (peior) 5,14 ; hirs (milium) $31 \mathrm{c}, 44$; rss in kerssen $27 \mathrm{~b}, 21$; kerssenpaum 51 d, 13; hyerssen (cervus) 52b, 34. 52c, 29. 52c, 34; hyerss 52c, 17; hierssen 52c,24. - Ulrich $\nabla$. Richenthal: $r s$ in Kürsiner 32. 182. 215; gehorsam 69; anderswa 51. 141; ars $109 ; r / s$ in Peler/shusen 72 u. ö. - Stretlinger chronik: rsch in berschaftig $\left.{ }^{3}\right)$, bresthaf $\ell$ : do tet

1) Entsprechend Heusler, Consonantism. d. ma. v. Baselst. s. 4 hat die urkunde no. 517 v. j. 1399 Hirlzbach s. 573 z. 15 , s. o. $\S 40$ anm. 2.

2) Hicrher gehört nicht erschatz ib., dazu das verb. vererschatzen ib., da es gewiss nicht vom verb. ersetzen abgeleitet ist (hierzu ursatz 'entgelt, recompensa'). Der zweite bestandteil ist sicher schaz 'geldsumme'. Verfehlt ist die zusammenbringung des ersten bestandteils mit her 'exercitus'; gewöhnlich wird er als êre 'honos' erklärt (DWB. 3, 954), eine leistung, die pro laude et consensu domini 'causa honoris' einmal beim wandel der hand vom nenen besitzer (käufer, erben) geleistet wird (laudemium) Z $8 p f$, Altert. d. d. reichs u. rechts 1, §33, $158 \mathrm{ff}$. Es bleibt aber gewiss, da bekanntlich die technischen ausdrücke deutschen rechtslebens misverständlicher ïbersetzung ins lateinische ausgesetzt waren, auch der vermutung raum gegönnt, dass wir in $e r$ - vielleicht $\hat{e} r, \hat{e}$ ' früuher, ehe, bevor' zu suchen haben. Man vgl. in der Stretlinger chronik eeschatz 40 neben erschatz 132. Demnach gäbe erschatz etymologisch den sinn, dass eine zahlung von seiten des meiers an den dominus vor der leistung des zinses $\mathrm{zu}$ geschehen hatte (?).

3) Für bersthaflig - vgl. birsthafl, Monumenta Zollerana 1, 510 (schwäb. linie), Lexer 1,350 - aus bresthaflig; keineswegs gilt Bächt- 
der berschaflig man nach der ler des alten 74,$2 ; r s$ in mörsel mörser $185,11 .{ }^{1}$ ) - Legende v. S. I dda v. 'Toggenburg (Alcm. 12,173 ff.): rsch in Raperschwil s. $176 ; r s$ in hirs s. 175. - Prologus zum elsäss. Parzival: rsch: uförschen v. 168. - Elsässischesarzneibuch (Al. 10, $219 \mathrm{ff}$.): $r s$ in Kirsen.

B) Ingold, Goldenes spiel: rsch: geherschot 6 (doch rs: person); anderschwa $58^{2}$ ); auch das $s$ des nentrums des geschlechtlichen pronomens wird sch geschrieben nach $r:$ pricht ersch alles 53 ; ich nill dirsch sagen 68. - Con rad von Wein B berg: $r s c h, r^{2}$ sch $\left.^{8}\right)$ : mir umb bürzschet zu Ermeln ij gulden 70,13 (bürschet, burschat halbseidenes zeug); rss: kürssen 13 (2). 26. 45. 47; kürssner 30. 52 (2); rs: kürsner 26. 5i. 88; kirsner 26; hans von hirshorn 73 ; Wickershein 52 (3); Otterswiller 68. Hermann v. Sachsenheim, Mörin: rsch: nur in murschel v. $3535 \mathrm{ff.:}$ 'nauch tülschen sillen manigvall sa/s wir zuosammen über tisch. Da truog wan her die murschel frisch, des allererslen rösch und wi/s'. Für Berschen (gen.) v. 3369 wird wohl Bertschen oder Berschen (adj. auf -isch) zu lesen sein; rs: wirs v. 42; kürsin 2881; anderswa 2985. K a ufringer: rs: hirsen 5, 633; kürsen 10, 75. 15, 17. 68; wirser 11, 99. 193. 12,117; ars 13, 238. 241 (: wars) ib. 333; rss: hirssen 5, 592. - Georg s von Ehingen reisen: rsch: perschon: er ist och von der perschon gantz wolgeslalt und lidmessig gewesin $\mathrm{s.} 4 \mathrm{z} .2$; bey des fürsten perschonen 8 z. 9. 38 ; $r s$ : forderst 4 ; fürsten 8 (also wenn $t$ folgt); $r s z$ in andersz s. 1. - Reimchronik d. Johannes $\mathrm{Kurz}^{4}$ ): rsch: Urschin v. 1. 2. 65. 194. 250. 478. 1010; Ürschin 264. 270. 319. 403; Kelerschwang 675.5) - Meichssner, Orthographie: rsch: Der hirsch würdl gebürscht 7s. fdph. 13,369 .

II. Im bairisch-ð̈sterreichischen.

1. Ans urkunden:

§ 43. Hyrs des Rodlers purchlehen, Schaup der Hyrs 1329, Urkb. d. l. o. d. E. 8. 554 ; Ott Wirsinch 1304, Urkb. d. l. o. d. E. no. 493; Chersperger sebr häufig, z. b. 1306 ib. 4, 515. 1327 ib. 5, 488 etc.; Cherspach 1325 ib. 5, 413 (8); Wyersing von Pottendorf 1335 ib. 6, 187; bürsen: wir schullen auch von unser bêrsen raichen jorlich .. ain phund phenning 1361 ib. 8 8. 10; rsch: vielleicht gehört Murchenhof (bei St. Peter in der Au N.OÖ.) 1360 ib. 7 s. 102 hieher.

holds erklärung in der anmerkung zn diesem worte: berschaftig umstellt aus breschhaftig.

1) hirz (cervus) 3, 19 u. s. o. wurde ebensowenig hier wie bei andern denkmälern berücksichtigt, wenn es mit $z$ oder gar mit $t z$ geschrieben war.

2) Hierher vielleicht anch noch verluscht $50<$ verlurscht $<$ verlurst.

3) Diese schreibung zsch für sch hat dieses denkmal auch im worte dülzsch deutsch s. 7 .

4) Aus Irsea (im bair. Schwaben bei Kaufbeuern).

5) Doch auch Ramschwang v. 600 neben Ramswang 449. 
2. A us literarischen denkmälern:

$\$ 44$. Hadamar v. Laber: rs: birs 43,2 ; birsen 46,1 . 510,5 ; gebirset 426, 2 ; birscer 543. - Oswalt v. Wolkenstein: rsch: merschy (merci frz.) 57,1,12. - Schiltberger: rs: hirsen $6 \mathrm{i}, 7 ; r / s:$ ander/swo 102, 31; rss: Wurssa 8,11. - 'Tucher: rs: kürsner 151,12; kursner 155, 9. 266, 20; morser 289, 5; Hans Morser 162,18; r/s im namen Hir/svogel 159, 30 etc. sehr häufig; Her/spruck 252, 25. 34; rss: hirssen 160, 22; Hirsselgasse 144,8(0̈); rsch in erbforschter 93, 8; hirschen 123, 16. 257, 16. - Tetzel hat s. 170 rs in hirs (milium).

III. Im mitteldeutschen.

$\S 45$. Buch von guter speise: rs: mursel 28 ; morsel 30 ; kirsen s. o.; kirsenmus 9, 82; hirse grülze 47; mörser s. 0 .; bersich (fisch, vgl. 8chm. 1, 201) 55. 62. - St olle, 'Thüring.-erfurtische chronik: rsch: zwene wagen mil ruchem wis/sen korschen wergk s. 107, 24; r/sch: geher/sche (inf.) uber land und lute 4,10; rssch: regeren (regieren) und hersschen 77,$10 ; r s$ in personen, personlich, torsle, fursten; $r / s$ in ander/s 36 ; Inger/sieuben 8. 0. - Bei Luther wechselt rs mit rsch bei hirsch (Franke, Grundzüge § 89). - Aus Schlesien weisen Pietsch-Rückert, Entwurf 8. 144 folgende rsi/h nach: herschin Psalterium per hebdom., 2. hälfte des 14. jh.; hershin abschrift einer psalmenübersetzung in pghs. 1 duod. 26, vollendet 1340; vurschte princeps, handschrift des Nicolaus v. Cosel, aus dem anfang des 15. und ende des 14. jh.; kurschner neben kursener im Cod. dipl. Silesiæ 8, 79; zu dem yrschten mal (2), ja sogar dyrsch $=$ dir ez Scriptores rerum Silesiacarum 6, 18 .

$\S 46$. Hieran mögen sich nachweise frluber $r s c h$-schreibung, welche die wörterbucher liefern, anreihen:

herschen: dem da herschel') met unde win Wälsch. gast $\nabla .4290$; wan in ze herschen gescliht über di siben ib. 9068; herschen (hershin) Fundyr. 1,376 a bei Conr. v. Heinrichau 1340 (DWB 4,2. 1155 a); herscher: Berth. 4169. Myst. 2,639. 1. 2. Walther v. Rheinau, Marienleben 274, 36 (s. Lexer 1, 1262 u. 1263); herschnier (mhd. hersenier subst. n.): duo herschnier 1316, Freys. salbuch f. 29 b (Schmeller 1, 1166); hirschgenige im mitteld. arzneibuch (Fundgruben 1, 3i6) a. d. 14. jh.; kurschen Cod. dipl. Sil. 3,122 ${ }^{2}$ ). Korschen Rothe, Diiring. chronik 99, der auch 430 hirschen herschen und 674 hirschte hat; murschel mhd. mursel, Heldenb. B 220, 32 (Lexer 1, 2254); morsch: daz alle sîn hût und sîn vleisch zumorschtt was D. Myst. 1, 185, 16 (14. jh. md.; DWB. 6, 2590; zer sch mhd. zers Cgm. 589 (v.j. 137i) hinterdeckel, Cgm. 713 (จ.j. 1476) f. 7,17 (Schmeller $2,1152)$.

1) So die handschriften A um 1300 und G 1340; Ruickert setzt herscht ein.

2) V. j. 1387 DWB 5, 2821 : mit einir kurschin. 
§ 47. Die frubesten rsch-schreibungen (anderschwa s. 253, herschen Meinauer naturlehre 253, Psalterium 255, Psalmenubersetzung ib., Wälsche gast ib., Conrad v. Heinrichau ib., herschnier Freys. salb. ib.) berechtigen uns, fur diese zeit auch die entsprechende aussprache anzunehmen und spätere $r s$ nur als historische schreibung aufzufassen. Zwei weitere stlitzen für diese aunahme bieten einmal das vorkommen der schreibung $r s$ für etymologisches $r s c$ (vgl. den analogen fall menslich für menschlich § 35, 7. b. Elisabeth 5032 gar unerforsbere sint godes ordenunge und Stolle \&. 42 erfor/ser: der selbe Jude was ern Apels unnd ern Bossen Vitzthum, des jungen hern rethe, heymelicher rath, vnnd alle ore heymelkeit eyn u/streger vnd erfor(ser), zum andernmal reime von wörtern mit ursprünglichem $r s$ auf solche, deren rsch auf $r s c$ zurtuckgeht (es liegt in der natur der sache, dass sich nur wenige finden lassen): örsch equus: tors stultus; Heinrich v. Beringen, Schachgedicht v. 2064. 9316 und rossen: gedrvschen Ottokars Reimchronik v. 60709 (letzteres glitige mitteilung des berrn professor Seemüller mit der bemerkung: 'die lesart ist sicher'). Wir können demgemäss diesen lautwandel als im 13. jh. vollendet auffassen.

III.

$\S 48$. Der wandel eines $s$ zu $\check{s}$ begreift sich wohl am leichtesten im zuletzt besprochenen falle. Denn das $r$ (zumal das stimmlose) hat grosse physiologische verwantschaft mit dem š. Sweet, A Handbook of Phonetics 39 beschreibt anschliessend an Bell das $\check{s}$ folgendermassen (Sievers $\S 152 \mathrm{~b}$, anm. 4): 'das $s$ ist dem $s$ sehr ähnlich, hat aber mehr von dem point-element, d. b. stärkere beteiligung des zungensaumes; dies hat seinen grund in der annäherung an stimmloses $r$; das $\check{s}$ ist in der tat ein $s$ das auf dem wege zu stimmlosem $r$ angehalten ist. Dies geschieht, indem man die zunge aus der $s$-lage ein wenig zurlickzieht und mehr nach oben wendet, was den zungensaum mehr in action bringt' (vgl. ubrigens auch Sievers s. $106 \S 12$ b.)

Schön stimmt zu dieser beschreibung des $\check{s}$ die tatsache, dass $r$ vor $d, t, z$ am Inn, Lech und an der Salzach geradezu wie $\check{s}$ lautet: fušt fort; kušz kurz u. dgl., vgl. Schmeller, Gr. §661. BWb. 1, 139. 1155. DMa 2, 344. 4, 44. Weinhold BG. § 155. 
Wenn wir abgesehen von den oben s. 252 erwähnten wörtern den wandel von $r s$ zu $r s ̌$ in der 'schriftsprache' nicht vorfinden, so haben wir darin - abgesehen von fremdwörtern wie börse, discurs u. dgl. - das resultat mannigfacher ausgleichungen $\mathbf{z u}$ sehen, die immer eintraten, wenn nach dem $r$ ein mit $s$ beginnendes wandelbares element (suffix) auftritt, z. b. -st der 2. sg., des superlativs, $-s$ des genitivs, die suffixe $-s c$, -sam u. dgl.

$\S 49$. Schwieriger ist das $\check{s}$ in den consonantenverbindungen zu begreifen in denen es als erster bestandteil auftritt.

Eine assimilatorische einwirkung der folgenden consonanz ist, da wir es mit dem wandel eines $s$ zu $\breve{s}$, d. h. mit dem wandel der tonlosen dentalen (alveolaren) spirans $\mathrm{zu}$ einer tonlosen spirans zu tun haben bei der jedenfalls die zungenarticulation stets weiter rickwärts liegt als beim $s$ ('mindestens 1 cm.', Gritzner s. 220), ohne weiteres begreiflich bei lauten, die entweder die gleiche articulationsstelle wie das $\grave{s}$ besitzen oder eine weiter rückwärts liegende.

§ 50. Sicher gilt diese erklärung also bei šk für sk in fremdwörtern, wie es die mundarten bieten. Also in wörtern wie: škandal (hierbei ist mit ausnahme der uns interessierenden $\check{s}$ die phonetische schreibung vermieden, da diese aussprache für das ganze obd. sprachgebiet gilt), škarnitzel dute; diškurirn reden, diškurs rede, streit, s. m. g. (vgl. Schm. 1, 549), muškel, mušketen muskete, muškateller muscateller (uber die letzten wörter vgl. Nagl, Roanad s. 200 zu v. 2377).1)

1) Ein secundärer vorgang ist es, wenn das $k$ dieser verbindungen, durch das $\dot{s}$ palatalisiert, weiter nach vorne geschoben als palatales $t$ erscheint, z. b. štarnizl 'papierdüte', ital. scarnuzzo (Schm.), štattel aus ital. scatola 'schachtel' (Schmellers Mundarten, nachträge hierzu in Herrigs Archiv 37, 374); śtuapiau 'skorpion' (Nagel a. a. o.). Für das alter dieser entwicklung scheint es zu sprechen, wenn eive hohenzollersche urkunde (kloster Wald) $\nabla$. j. 1430 den tag ihrer ausstellung mit den worten 'nach sant St olastican tag' angibt; vielleicht ist auch damast $\mathrm{n}$. (zu grunde liegt der name der stadt Damascus) hierherzuziehen, doch sprechen ital. damasto und nl. damast dagegen, wenn diese nicht deutsche lehnwörter sind. - Belege für schk aus früher zeit s. 0. § 29. - Eine parallele zu der verschmelzung der alten $s k$-verbindungen bietet das mundartliche neuširig 'nengierig', entstanden aus neusgierig (Vilmar, Kurhess. idiotikon 283. Schmeller 1,1711. Ronsdorfer ma., Zs. fdph. 19, 362). Hält man zu obiger erwägung die tatsache, wie oft sich die palatalen š-laute 
§51. Wahrscheinlich gilt die annalume einer solchen assimilierenden einwirkung des folgenden consonanten bei der erklärung einer grossen zahl der inlautenden -št der alemannischen mundarten. Denn es wird wohl nicht als reiner zufall zu betrachten sein, wenn ein grosser teil dieser wörter nach declinationsschemen geht, die ursprünglich in gewissen casus ein $i$ in der endung besassen. Ich erinnere hier nur an wörter wie mhd. ast, bast, brunst, brust, ernst, vâst, geist, gerüste (jodecl.), geswulst, gespenste n. (jo-decl.), daneben gespanst, gespenst fem., gunst, last, list, lust etc. etc. In allen den fällen, in denen bei diesen wörtern und ihren ableitungen palatalisierung des stammsilbenvocals ('umlaut') - wenn er nicht schon selbst ein palataler war - eintrat, hätten wir auch das $̌ s$ als eine wirkung des folgenden $i$ zu betrachten, wie man ja auch jetzt die palatale affection des vocals durch folgendes $i$ als durch die zwischenstehenden consonanten vermittelt erklärt, welche zunächst palatale färbung ('mouillierung') annehmen mussten, und dann ihrerseits die palatalisierung des vocals bewirkten (vgl. Sievers, Phonetik ${ }^{3} 237$ f. Braune, Ahd. gr. § 51 anm. 1).1)

$\S$ 52. Ausser nach $r$ wäre somit št entstanden: a) ursprtunglich wechselnd mit st bei der $i$-declination (gast pl. gešte) und im präs. der sw. verba I, b) ohne wechsel, durch bildungssuffix veranlasst, z. b. nomina mit $j$-suffix wie gerïšte; adjectiva wie vešte, abstracta auf $-\hat{\imath}$ wie rešte ruhe, rast, wobei bei den

im klange den palatalen ch-lauten (ich-lauten) nähern (Sievers, Phonetik $\S 15$ anm. 5) - man vergleiche das hinterwäldlerische gweisch 'geweih' (Birlinger, Al. spr. s. 134. Weinhold, Dialektf. s. 82) - so hat man sich die entwicklung des $s c \mathrm{zu}$ s vielleicht folgendermassen vorzustellen: $s k>\grave{s} k>\grave{s}-c h>\grave{s}$. Hierher zu stellen ist die eigentlimlichkeit der Kuhlaer mundart (Regel s. 73), für stammauslautendes $s$ vor dem diminuierenden -chen $\grave{s}$ zu bieten, z. b. glešchen 'gläschen', müšchen 'mäuschen', Lišchen 'Lieschen', gänšchen, henšchen 'Hänschen' etc.

1) Natiurlich musste der vor dem palatalvocal stehende consonant, das $t$, seiner articulation nach ein palatallaut sein (vgl. Kruszewski, Internation. zs. 2, 267). - Ueber $i$-ableitung bei mhd. gester, swester 8 . Kauffmann, Beitr. 13, 393. 14, 163 gegen Luick ebenda 13, 588 f. - Die umlautswirkung des $\grave{s}$ aus $s$ vor palatalem $t$ fände eine schöne parallele

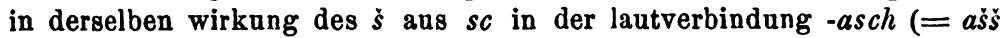
$<a s c)$, das regelmässig in den alem. mundarten zu ęšs wurde, z. b. ęšša 'asche', węššse 'waschen'. Vgl. Brandstetter \$ 19. Heusler, Germ. 34, 117. 
unter a) angefihiten das št durch ubertragung verallgemeinert worden wäre. Für eine gesonderte entwicklung dieser inlautenden št scheint es auch zu sprechen, dass sich zeugnisse, welche die $s$-aussprache in consonantenverbindungen beweisen, am ehesten bei st finden (vgl. oben $\S 36$ ).

$\S 53$. Schwieriger ist es, mit den andern $\check{s}$-verbindungen zurecht zu kommen. Denn bei diesen ist eine assimilierende wirkung des folgenden consonanten nicht recht glaublich, schon aus phonetischen grtinden, da ja bei den labialen $(p, w, m)$ die zunge gar nicht articuliert und die dentalen $(t, n)$ dieselbe alveolare articulationsstelle wie der ursprüngliche laut, das $s$, besitzen, und sie ist auch wegen der inlautenden bewahrten $s t, s p$ des mitteldeutschen bezw. der st im bair.-österreichischen vollkommen ausgeschlossen.

Man kann aber auch nicht vermuten, dass eine verschiedenartige silbentrennung an diesen verschiedenheiten zwischen mittelund oberdeutsch schuld trlige, sonst mussten wir für das bair.österreichische einmal etwa ka-špar und zum andern etwa klo-ster ${ }^{1)}$ ansetzen.

$\S 54$. Lässt sich nun das $\check{s}$ in diesen verbindungen kaum durch einwirkung des folgenden consonanten erklären, so war vielleicht ein vorhergehender laut die ursache dieses lautwandels, und der wäre aus phonetischen gründen und wegen des oben 8. 252 ff. auseinandergesetzten das $r$.

Ės wären demnach unsere sch-verbindungen im wortanlaute sandhierscheinungen, hervorgerufen durch die stellung der wörter mit ursprunglichem $s l, s m, s n, s p$, st und $s w$ nach wörtern die auf $r$ endigen. An solchen mit $-r$ schliessenden wörtern ist aber unsere sprache sehr reich. Hierher gehören: die substantiva auf -er; die adjectiva im nom. sg. m. starker declination, im gen. sg. und pl. st. decl. fem., im dat. sg. f. st. decl. (eine kategorie, in welche die so häufig gebrauchten wortclassen wie der artikel und die demonstrativpronomina gehören), die comparative, die personalpronomina mit ihren: meiner, mir, wir, unser; deiner, dir, ihr, euer; er, seiner, ihrer, ihr; die praepo-

1) Singulär steht da -št in huaštar 'tussis' (Nieder-Oesterreich), vgl. Nagl s. 201 zu v. 231. 
sitionen: ausser, für, über, unter, vor u. s. w.; die praefixe: er-, ver-, zer- u. s. w.

Und an diesem anschlusse an ein vorausgehendes mit $r$ schliessendes wort trüge die dem $s$ folgende consonanz die schuld; sie trennte das $s$ von dem träger des silbenaccentes, von dem ibr folgenden sonanten, weil ihr eine geringere schall, fulle innewohnt, die bei $p$ und $t$ bis auf denn nullpunkt sinkt; diese bedingen sogar eine pause!

$\S 55$. Auf dieser tatsache der lautphysiologie (Sievers, Phonetik ${ }^{3} \S 25$. 26. 27) fussen ja auch die vergleichungen der indogermanischen sprachen, bei welchen wörter des typus $s+$ consonant (im anlaut) mit wörtern, die das $s$ entbehren, zusammengebracht werden; vgl. Brugmann, Grundriss 1, §55 ff. G. Meyer, Griech. gr. ${ }^{2} \S 245 \mathrm{ff}$. und neuestens K. F. Johansson, Beitr. 14, $289 \mathrm{ff}$. Zur illustrierung seien die sichereren fälle angefuhrt:

Für sl-l: Ir. slath stange, ahd. latta f. latte, bret. laz. - Got. ahd. as. slahan, anord. slá, ags. sléan, aber gr. $\lambda \alpha x i \zeta \omega$, lat. lacerare. Got. slaupjan, ags. slúpan, nord. got. sliupan, ahd. sliofan, lit. slübnas schwach gegen lat. lûbricus schlüpfrig; wz. sli glatt, schlüpfrig sein, ahd. slimen glätten, mhd. slim, an. slím, aber lat. limare feilen, glätten, lima feile, levis, gr. $\lambda \varepsilon i \sigma_{0}$ glatt.

Für $s m-m$ : Ausser gr. $\sigma \mu \iota x \rho o ́ s$ neben $\mu \iota x \rho o ́ s, \sigma \mu v \dot{\rho} \rho \alpha, \mu v \dot{\rho} \rho \alpha$, $\sigma \mu \tilde{\tau} \lambda \alpha \xi, \sigma \mu \tilde{\tau} \lambda$ os taxusbaum, $\mu \tilde{\imath} \lambda \alpha \xi, \mu \bar{\tau} \lambda o \varsigma, \sigma \mu \tilde{\eta} \varrho \iota \gamma \xi$ borsten, $\mu \tilde{\eta} \varrho \iota \gamma \xi$. - Ahd. smelzan neben gr. $\mu \dot{\varepsilon} \lambda \delta \omega$, dazu ags. meltan, an. maltr (adj.) verfault, ahd. mhd. mulz einschmelzend, weich, schlaff. - Got. smals, ahd. smal, ags. smael, as. smal klein, gering, aber asl. malü klein, gr. $\mu \tilde{\eta} \lambda \alpha$ kleinvieh, altir. mil tier (dieselbe bedeutung hat anord. smali kleinvieh; vgl. auch ahd. smalanóz, smalaz vihu kleinvieh). - Gr. $\mu \varepsilon \iota \delta \alpha^{\prime} \omega$ lächle (doch $\varphi$ $\lambda o \mu \mu \varepsilon \imath \delta \dot{\eta} s)$, aber lett. sméju, asl. smě-jq sę lache, ai. smáy-a-lè, dazu ahd. smielen.

Für sn-n: Av. snaçžathi, air. snechta, got. snaivos, lit. snẽgas, asl. snĕgŭ schnee gegen gr. $v \varepsilon i ́ \varphi \varepsilon \iota, v i \varphi \alpha$ (aber $\alpha^{\prime} \gamma \dot{\alpha} \nu v \iota \varphi o s$, lat. ninguit. - A hd. snuor, air. snáthe faden gegen lat. neo, gr. $\nu \dot{\varepsilon} \omega \nu \tilde{\eta} \mu \alpha$ faden, aber "̌̀v nebat. - Ahd. snura f. schwiegertochter, ags. snoru, afr. snore, an. snor, sner zu aind. snušâ, asl. snŭcha gegen lat. nurus, gr. vvós. - Ahd. snabul m., ndl. snavel schnabel, rüssel, sneb schnabel, afr. snavel mund gegen ndl. neb f. schnabel, ags. nebb schnabel, gesicht, an. nef n. nase (neben snafor feinriechend), dazu auch das germ. lehnwort ital. niffo schnauze, rüssel.

Für $s p-p$ : Got. speiwan, ahd. spîvan speien zu lit. spiáuju speie, at. spuo gegen gr. $\pi \tau \bar{v} \omega$. - Ai. spáç späher, wächter, lat. -spicio, ahd. 
spehôn spähen, anord. spakr verstïndig gegen ai. páçyämi sehe, asl. paziti attendere (reflex. mit se cavere). - Got. spinnan, ahd. spinnan spinnen gegen lit. pinti flechten (pántis strick) und asl. pęti spannen, von der darin enthaltenen wz. pen- lat. panmus stückchen tucb, lappen, asl. o-pona vorbang, ponjava fem. segel, zu welcher sippe auch unser fahne (got. fana tuch, zeug, lappen, ahd. fano tuch [ougafano schleier, halsfano halstuch, gundfano fahne] masc. gehört. - Idg. wurzel sprek, spreg gegen prek, preg in isl. sprekla, schwed. spräkla kleiner fleck, mhd. sprëckel (sprünkeleht adj. fleckig) gegen engl. to freak sprenkeln,

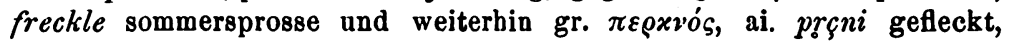
bunt.

Für $s t-t$ : Ai. sthágāmi verdecke, verhülle, gr. $\sigma \tau \dot{\varepsilon} \gamma o s$ dach, lit. sloga-s dach, aksl. o-stegŭ toga gegen gr. $\tau \dot{\varepsilon}$ yos dach, lat. lego, aisl. pak, ahd. dah dach. - Got. stáutan, ahd. stôzan stossen gegen ai. tudámi stosse, lat. tundo. - Gr. $\sigma \tau i \gamma \mu \alpha$ stich, punkt zu $\sigma \tau i \zeta \omega$ mit einem spitzen werkzeuge flecken machen, lat. instigare anstacheln, reizen, ahd. stëhhan stechen gegen ai. tij scharf sein, schärfen (tigmá spitzig, scharf). - Ai. sthûra gross, mächtig, zend. staora zugvieh, got. stiur, ags. stéor, ahd. stior stier gegen asl. turŭ stier, gr. $\tau \alpha \tilde{v} \varrho v_{S}$ (lat. taurus), an. pjorr (dän. tyr, schwed. tjur); aber anord. stórr, ahd. stûri adj. gross, mächtig, air. tarb. - Ahd. stinchan stinken gegen gr. $\tau \alpha \gamma \gamma o ́ s$ ranzig. - Lit. strázdas

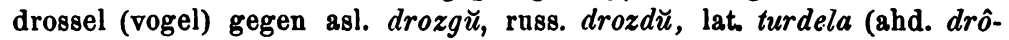
scela, baier. drôschel), mhd. drostel, an. prostr masc. - Ahd. drozza, ags. protu fem. kehle gegen mhd. strozze, andd. strota kehle, luftröhre. - Mhd. nhd. strotzen, engl. strut anschwellung, schwellen, gegen anord. pruitinn geschwollen.

Das fehlen des ursprunglichen $s$ der grundformen in manchen indog. sprachen wird als wirkung eines sandhi zu erklären sein, der durch die dem $s$ folgende consonanz erleichtert wurde. Dieses moment der erleichterung des sandhi ist aber, wenn auf das $s$ unmittelbar der sonant, der träger des silbenaccentes, folgt, nicht vorhanden.

§ 56. Ein anderes moment der erleichterung des sandhi liegt in dem stärkeverhältnis der einzelnen silben zu einander, da sich ja minder betonte silben einer stärker betonten silbe unterordnen und sich so zu einer grössern phonetischen einheit, dem sprechtakte, zusammenfügen. Die starken silben in solchen sprechtakten bilden die stärksten silben der wörter, und das sind die stammsilben. Hiernach (dazu vgl. Sievers § 33) wurden die $s$ und $\check{s}$ im anlaut der wörter und silben zu erklären sein. Es blieb nämlich I) (stets vorausgehendes $r$ vorausgesetzt) $s$ vor dem sonanten der starken silbe des sprechtaktes, also im 
anlaute der stammsilben ${ }^{1}$, wurde aber II) zu $\check{s}$ in anlaute der mittelstarken und schwachen silben desselben typus, also a) in nebensilben und b) bei encliticis.

So haben wir:

Ad I. sagen, sehn, singen, suchen u. dgl.

Ad II. a) Die oben s. 252 erwähnten wörter in ihren zweisilbigen formen, wenn dem ursprtinglichen $s$ ein vocal folgt ${ }^{2}$ ) (hierber kann man auch rechnen die wörter des typus $\grave{s}+$ consonanz, vgl. oben § 54); diesen schliessen sich an mundartliches ghoršam, koršam gehorsam (besonders häufig in der grussformel kšamster diener, šamster diener fǜ gehorsumster diener) Schmeller, Ma. §651. D. Ma. 3, 107. Bavaria 3, 209. Albrecht s. 15. Bronisch s. 129. Weinhold, Dialektf. 80) und uršach (s. Birlinger, Al. spr. 132: urschach legende des hl. Aurelius, hs. d. 17. jh.).

b) Hierher gehören mundartliche verbindungen wie unašic, adv. f. unter sich, 'nach unten'; fürši adv. f. für sich, 'vorwärts'; hinterši adv. f. hinter sich, 'rückwärts'; hinterš̌ifürši adv. f. hinter' sich für sich, 'vorwärts-rtickwärts' also: 'verkehrt' (Winteler, C, cap. I, § 4. 1 b; Schmeller, Ma. §652. Bavaria 3, 209. Handschuchbeimer glossar. Mundart des burggrafenamtes, Herrigs Archiv 43, 179. Krassnig s. 32). -- Ferner: ši, še mundartlich für das personalpronomen sie in verbindungen wie: war še 'war sie', hör še 'höre sie', über še 'tiber sie', gib mir še, dir še 'mir, dir sie'. Schm., Ma. § 651. D. Ma. 3, 107. 129. Regel 73. Hierher ziehe ich auch das thtiringische und hennebergische

1) Mit scheinbarer ausnahme von mundartlichem úršach ur'sache, das aber mit seiner betonung auf ir- im gegensatze zu etwa ersétzen das verhältnis um so klarer darstellt.

2) Die mundarten sind, wie schon erwähnt, an solchen wörtern reicher. Mit $\dot{s}$ lauten noch: borse, ferse, hirse, mörser, pfirsich, lirsel Ursula, vers, wirsing; dazu: farce füllsel, force stärke. Dazu noch die wörter mit -rst wie durst, forst, wurst, denen noch verlurst Neujahrsspiel v. 336, Stretlinger chron. 25, ägerst 'elster' schwäb. Schw. Wb. 1, 48 zuzutügen sind. Durch methatesis kommen hinzu die mundartlichen wörter für brust, krusle, presse Krefelder mundart, D. Ma. 7, 50, kresse Regel s. 64. Zndem die genitive auf $-r$ auslautender wörter mit $s$, z. b. bruder $\dot{s}$, und die verbindung tbensolcher wörter mit dem nom. acc. sg. des geschlechtigen pronomens, z. b. dir š dir es, nicht nur in thüring. ma. Vgl. Winteler C. I, §4,1 b; Schmeller Ma. §652. Ueber ršt in der flexion und beim superlativ s. unten $§ 48.59$. 
goirše 'gar so' in wendungen wio goirše šlömm 'gar so schlimm', goirše gärn' 'gar so gern', vgl. D. Ma. 3, 129. Regel 73.

$\S 57$. Wir können also ganz allgemein die regel aufstellen: In der stellung nach $r$ wurde $s$ zu $\check{s}$, wenn ihm unmittelbar ein den hauptaccent nicht tragender laut folgte.

Unsere lautverbindungen $\check{s}+$ consonant wären also entstanden, wenn dem ursprüglichen $s+$ consonant ein $r$ vorhergieng; ein satz, wie dẹr vatẹrš pilt 'der vater spielt' ist in seinen taktverhältnissen conform einem dęs doklorš rech [nung] 'des doctors rechnung'. Die nach $r$ entstandenen $\check{s}+$ consonant-verbindungen hätten dann ihr geltungsgebiet erweitert. Diesen wandel möge folgendes schema veranschaulichen:

I.

ich spile
du spilst
er spilt
wir spiln
ir spilt
si spiln

II.

ich spile

du spilst

er špilt

wir špiln

ir špilt

si spiln
III.

ich špile etc.

(durchaus šp)

I stellt die verhältnisse vor, II während und III nach der wirksamkeit des gesetzes dar.

Da nun $-r s ̌ s ~>r s$ mindestens fur das 14. jh. anzusetzen ist, so wäre auch die wirksamkeit dieses sandhi in jene zeit zu verlegen, und damit stimmten auch die oben nachgewiesenen schreibungen von sch + consonanz. $\left.{ }^{1}\right)$

§ 58. Gegen diese hypothese lässt sich aber folgendes geltend machen:

1. Der mangel eines directen nachweises des sandhi aus

1) Folgende zusammenstellung möge eine übersicht von diesem gesichtspunkt (sch nach $r$ ) ausgehend gewähren. Hierbei sind kürzere denkmäler, aber auch urkunden und weistümer, naturgemäss nicht in betracht gezogen, und von den grössern denkmälern die nicht übergangen worden, die untermischt $s$-oder sch-schreibung in irgend einer der hiehergehörigen consonantenverbindungen aufweisen : 
den denkmälern. ${ }^{1}$ ) Aber dieselbe trägheit der orthographie finden wir auch bei den wörtern mit $r s$.

2. Dass wir auch sonst nicht nur nach $r$ inlautend $\check{s} t, \check{s} p$, šw u. s. f. besitzen.

\begin{tabular}{|c|c|c|c|c|c|c|c|c|}
\hline \multirow{2}{*}{ Denkmal: } & \multicolumn{2}{|c|}{ sl: schl } & \multicolumn{2}{|c|}{ sm : schm } & \multicolumn{2}{|c|}{$s n: s c h n$} & \multicolumn{2}{|c|}{$s w: s c h w$} \\
\hline & übh. & n. $r$ & übh. & n. $r$ & übh. & n. $r$ & übh. & n. $r$ \\
\hline & $9: 1$ & $5: 0$ & \multicolumn{2}{|c|}{$s \boldsymbol{n}$} & $S: 1$ & $2: 1$ & \multicolumn{2}{|l|}{$s i v$} \\
\hline \multirow{3}{*}{ 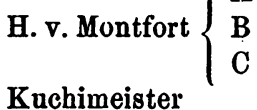 } & $4: 49$ & $2: 7$ & \multicolumn{2}{|c|}{$11: 2 \mid 5: 0$} & $1: 16$ & $1: 7$ & \multicolumn{2}{|c|}{$s n$} \\
\hline & $0: 4$ & $0: 1$ & \multicolumn{2}{|c|}{$1 \mathrm{sm}$} & \multicolumn{2}{|c|}{1 schn } & \multicolumn{2}{|c|}{$s w$} \\
\hline & $22: 2$ & $19: 0$ & $1: 2$ & - & \multirow{2}{*}{\multicolumn{2}{|c|}{$\begin{array}{l}\text { schu } \\
\text { schu }\end{array}$}} & $26: 13$ & $4: 1$ \\
\hline Ingold & \multicolumn{2}{|c|}{ schl } & \multicolumn{2}{|c|}{ schm } & & & $23: 40$ & $2: 4$ \\
\hline \multirow{2}{*}{$\begin{array}{l}\text { Mörin } \\
\text { Kaufringer }\end{array}$} & $27: 56$ & $4: 4$ & \multirow{2}{*}{\multicolumn{2}{|c|}{$\begin{array}{c}18: 12 \mid 0: 1 \\
\text { schm }\end{array}$}} & \multirow{2}{*}{\multicolumn{2}{|c|}{ 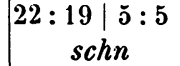 }} & $106: 44$ & $15: 4$ \\
\hline & $64: 22$ & $9: 6$ & & & & & $104: 15$ & $13: 7$ \\
\hline \multirow{2}{*}{$\begin{array}{l}\text { 0. v. Wolkenstein } \\
\text { Schiltberger }\end{array}$} & \multicolumn{2}{|l|}{ sl } & \multirow{2}{*}{$77: 10$} & $21: 1$ & \multirow{2}{*}{$\begin{array}{l}60: 2 \\
2: 11\end{array}$} & \multirow{2}{*}{$\begin{array}{c}23: 0 \\
1: 0\end{array}$} & $168: 4$ & $25: 2$ \\
\hline & $s c h$ & & & $1: 0$ & & & $14: 50$ & $0: 3$ \\
\hline \multirow{3}{*}{ Tucher $\left\{\begin{array}{l}\text { A } \\
\text { B }\end{array}\right.$} & \multirow{3}{*}{\multicolumn{2}{|c|}{\begin{tabular}{c|c|}
$159: 431$ & $19: 29$ \\
$30: 3$ & 2 schl
\end{tabular}}} & \multirow{2}{*}{\multicolumn{2}{|c|}{$27: 62 \mid 10$}} & \multirow{3}{*}{\multicolumn{2}{|c|}{$\mid$\begin{tabular}{c|c}
$34: 60$ & $16: 9$ \\
$4: 16$ & $3 \operatorname{sch} n$ \\
$\operatorname{sch} n$ \\
$\operatorname{sch} n$
\end{tabular}}} & $77: 14$ & $10 \mathrm{siv}$ \\
\hline & & & & & & & $s w$ & \\
\hline & & & $\begin{array}{l}\text { sch } \\
\text { sch }\end{array}$ & & & & $\left|\begin{array}{c}56: 65 \\
4: 88\end{array}\right|$ & $\mid \begin{array}{c}1: 2 \\
0: 18\end{array}$ \\
\hline
\end{tabular}

Die fälle, in denen unsere consonantenverbindungen nach $r$ auftreten (in der tabelle je die zweite spalte) sind vorne in der belegsammlung durch sternchen bei der seiten- oder verszahl ihres vorkommens gekennzeichnet.

1) Wenn auch demgemäss ein directer beweis aus den denkmälern nicht zu erbringen ist, so möchte ich doch die anfmerksamkeit auf zwei tatsachen lenken, die uns vielleicht noch leise spuren von der wirksamkeit unserer regel aufweisen. Erstens: unter den wörtern die mit $s n$ anlauten, nehmen eine hervorragende stellung hinsichtlich ihres gebrauches die verallgemeinernden pronomina und pronominaladverbia (swer, swaz, swie, swo etc.) ein. Diese wörter, die sich bis ca. 1400 im gebrauch behaupten (vereinzelt noch im 15. jh., so swas Kaufringer 8, 6, 10), finden sich regelmässig mit $s w$ geschrieben: ausnahmen sind sehr selten (man findet dieselben aufgezählt cap. $1 \S 32$, a). Der grund hierfür mag darin liegen, dass diese wörter, die immer zu anfang eines satzes gebraucht wurden, gar nicht unter unsere regel fallen konnten, $d$. h. die consequente $s$-schreibung in diesen wörtern bewiese indirect einen sandhi. - Dann erklärt sich auch ungezwungen, dass dem $s w$ gegenüber den $s l, s m, s n$ ein längeres leben gegönnt ist, wenn man eine beeinflussung der orthographie durch die $s w$ der verallgemeinernden pronomina und pronominaladverbia annimmt. Zweitens: tabellarisch sind nachfolgend die verbältnisse bei scheidung der mit $s w(s c h w)$ anlautenden wörter 'nach wortarten dargestellt: 
§59. Ein grosser teil der alem. inl. št fällt hier allerdings ausser betracht (s. oben § 51). Bei weitem der grösste teil der alem. št aber mussen als tibertragungen von wörtern in denen sie berechtigt sind (d. i. in der stellung nach $r$ ) aufgefasst werden. Es sind dies die š $\iota$ welche als flexionsendung oder suffix auftreten; hierher gehört vor allem das -st der 2. pers. sg. Ein waršt, wäršt, wiršt des verb. subst. lassen ein bišt (dem sich nach der proportion lauscht 2. sg. pr. v. verb. lauschen : lauscht 3 . sg. pr. = bišt $: x$ išt anschloss ${ }^{1}$ ), begreiflich erscheinen. Kurz dieses $s$ t, welches bei verben wie gebären, dörren, ehren, fahren, führen, begehren, hören, kehren u. ä. sich not-

\begin{tabular}{|c|c|c|c|}
\hline Denkmal: & $\begin{array}{c}\text { I } \\
s w: s c h w \\
\text { verbum }\end{array}$ & \begin{tabular}{|c|} 
II \\
$s w: s c h w$ \\
adject. (adv.)
\end{tabular} & $\begin{array}{c}\text { III } \\
s w: s c h w \\
\text { substantiv }\end{array}$ \\
\hline Kuchimeister & $14: 9$ & 一 & $12: 4$ \\
\hline Stretlinger chr. & $36: 2$ & $6: 0$ & $17: 0$ \\
\hline Richental & $14: 33$ & $7: 10$ & $\begin{array}{c}35: 22 \\
8 \text { swert }: 9 \text { schwert }\end{array}$ \\
\hline Ingold & $4: 7$ & $4: 13$ & $\begin{array}{c}15: 20 \\
12 \text { swert }: 10 \text { schwert }\end{array}$ \\
\hline Mörin & $47: 20$ & $27: 13$ & $\begin{array}{l}32: 11 \\
7 \text { swert }\end{array}$ \\
\hline Kaufringer & $30: 10$ & $23: 0$ & $\begin{array}{c}51: 5 \\
5 \text { swert }\end{array}$ \\
\hline Schiltberger & $1: 8$ & $7: 19$ & $\begin{array}{c}6: 23 \\
2 \text { swert }: 18 \text { schwert }\end{array}$ \\
\hline $\begin{array}{l}\text { 'Tucher } \\
\text { 'Tristrant }\end{array}$ & $\begin{array}{l}25: 8 \\
\text { schw }\end{array}$ & $\begin{array}{l}4: 0 \\
\text { schw }\end{array}$ & $\begin{array}{c}48: 6 \\
4: 41 \\
2 \text { swert : } 22 \text { schwert } \\
1 \text { swager: } 6 \text { schwager }\end{array}$ \\
\hline
\end{tabular}

Man sieht bei vergleichung der drei gruppen untereinander, dass schn beim verbum beliebter ist als beim adjectiv (adverb) und substantiv. Und dies kann vielleicht auch als eine spur des sandhi gedeutet werden, insoferne als bei dieser wortklasse eine häufigere, beziehungsweise festere verbindung mit auf $r$ auslautenden wörtern und partikeln (ich verweise nur auf die präfixe er-, ver-, zer-) vorkommt.

1) Das entgegengesetzte bietet die ma. an der mittleren Altmulhl in der 2. sg. verb. subst. is, 3 . ist, in der sonst $s t$ durch $\check{s} t$ vertreten ist (D. Ma. 7, 389). 
wendigerweise als flexionsendung der 2. sing. ausbildete, wurde als die 2. pers. charakterisierend empfunden und verallgemeinert. Auf ähnliche weise wäre auch das -št des superlativs, ursprünglich nur berechtigt bei wörtern die auf $r$ auslauten, so in superlativen wie eršte, ungeheueršte, teueršle, vorderšle u. a., auch auf anders auslautende wörter ubertragen worden.

$\S 60$. Dieser erklärung aber entziehen sich die andern inlautenden $\check{s}$-verbindungen des alem. bezw. bair.-österr. Es sind dies vor allem die wörter mit inl. šp (bei šw kommt wohl nur Ošwalt in betracht). In anbetracht ihrer geringen anzahl könnte man vielleicht lautliche analogie ${ }^{2}$ ) annehmen.

Dafür würde es sprechen, a) wenn sich in mundarten die sonst št bieten, etwaige st zeigen, und b) wenn sich in mundarten die sonst st bieten, etwaige št zeigen. Jene würden

1) Die alem. ortsnamen auf -schwil, -schwang liessen sich als übertragungen des suffixes von wörtern, in denen es regelrecht stand, erklären. Also nach Rapperschwil, Ganderschwil u. dgl. auch Attenschwil u. dgl.

2) Ueber das wesen der lautlichen analogie spricht sich Schuchardt, Ueber die lautgesetze s. $7 \mathrm{ff}$. folgendermassen aus: '(Andrerseits) lassen sich nicht selten erscheinungen bei denen durchaus begriffliche beziehungen im spiele sind, suf ideelle nebeneinanderstellung zurïckführen, und da können wir von einer niedrigeren ordnung von analogiebildungen reden. So begünstigt die hänfigkeit gewisser lantcomplexe die neubildung identischer (z. b. $i e ́=i e$ in ital. pièta) oder die häufigkeit eines gewissen lantwandels wird zur allgemeinheit. Ich habe vor langen jahren den gedanken geäussert, dass im ital. (und im romanischen taberhaupt) $i e, u o$ - vulgärlat. $e, g^{-}$ursprünglich, wie noch jetzt, in manchen dialekten, an ein folgendes $i$ oder $u$ gebunden war: vieni, buonu, buoni. Zunächst würde es durch begriffliche analogie ausgedehnt worden sein: viene, buona, dann aber auch ohne eine solche: pietra, ruota und formen wie bene, bove (plur. buoi), nove (gegeniiber nuovo) würden eben die letzten uneroberten plätze bedeuten'. Vgl. auch Easton im Am. journ. of philol. 5, 174. Hinzuweisen ist anch auf die analogien welche die fremdwörter bieten. Bei diesen sind auch lautsubstitutionen (so nennt diesen vorgang Gröber) zu beobachten, die ihre ursache darin haben, dass 'der sprechende mit denselben bewegungsgeftihlen, mit denen er seine muttersprache hervorbringt, auch das wort aus der fremden sprache erzeugt (vgl. Paul, Principien ${ }^{2}$ 8. 340. 341. Kruszewski, Intern. z8. 3, § 22, 2, § 30; gesetz der lautcombinationen ib. anm. 2, § 32 ; Intern. zs. 5, § 78. Jespersen, Intern. zs. 3, 194. 211. 212). 
dic noch uneroberten positionen darstellen, diese den beginn jener dort vollzogenen bewegung andeuten.

Zum ersten fall kann man vielleicht rechnen das part. prät. gewest der Handschuchheimer ma. (Lenz s. $54^{\mathrm{b}}$ ), die sonst inl. $\check{s} t$ hat. So auch in der Rheinpfalz (Bavaria 4, 242). Bei dem präteritum und participium der verba reisen, wissen, müssen u. dgl. mag - wenn nicht formen auf -et zu grunde liegen der $s$-laut der andern formen eingewirkt haben. Ferner könnte das unerklärte aister 'immer' der Schweizer ma. (Brandstetter, Geschfr. 38, 250) hierher gehören. Vgl. auch 8. 265 anm.

Zum zweiten falle wären zu rechnen die šp des bair.-österr. und die sonst sporadisch auftretenden št wie geweš $t^{1}$ ) 'verdorben' neben gewest (Bavaria 4, 242), das bereits erwähnte niederösterr. huaštn, ferner pašlur in der Ronsdorfer Ma. ${ }^{2}$ )

§61. Der dritte einwand der erhoben werden kann, sttitzt sich auf die verhältnisse in der ma. von Baselstadt. Es setzt nämlich die oben aufgestellte hypothese alveolares $r$ voraus, das dieser ma. aber nicht oder nur selten eigentümlich ist. Es herscht vielmehr neben dem zitterlaut des zäpfchens der stimmlose velare reibelaut ohne jede mitwirkung des zäpfchens vor, welcher oft zum reducierten laute ohne hörbares reibegeräusch wird (Heusler s. 82). Conform diesen verhältnissen heisst es auch in Baselstadt bi $\chi s$ f. der Birsfluss u. dgl. Daraus folgt, dass diese aussprache des $r$ eine ziemlich alte ist. ${ }^{3}$ )

Wie aber auch noch heute daselbst die aussprache des $r$ eine verschiedene ist (es kann nämlich nach Heusler a. a. 0. als reducierter laut ohne reibegeräusch eintreten im wortanlaut

1) 'Am Konigsdorf im Grabfeld (hört man) gewaschl, aber ist' Schmeller, Nachträge. Herrigs Archiv 37, 374.

2) Substitution der lautcomplexe begünstigt durch die gleichheit des ersten bestandteils und durch das übergewicht des complexes in den sich der alte wandelt, ist bei hašll neben hašpel (dazu das verb. haštnen, Schmeller Wb. 1, 1185) anzunehmen, wenn beide wörter identisch sind (ein zusammenhang mit hd. hast fem., hasten, die dem md. ndd. entstammen, Kluge, Etym. wb. 132 b; ist natürlich abzuweisen). - Auch auf die mundartlich oft vorkommende vertauschung von mišlel und mišpel sei hier hingewiesen.

3) Die belege aus den urkunden (s. oben) mit rsch in herschen und Birsch können an und für sich nichts gegen das alter dieser aussprache beweisen. 
und $z$ wischen zwei vocalen oder $z$ wischen vocal und folgendem sonorconsonanten auch in den wortanlautenden verbindungen $p \chi, \iota \chi, k \chi, f \chi, \check{s} \chi$, deren erster bestandteil gleichzeitig zur reinen lenis herabsinkt; niemals jedoch im freien auslaut oder vor stimmlosen consonanten) so wird wohl schon fruher furr $r$ irgend ein unterschied je nach der erwähnten stellung bestanden haben. Andrerseits ist es ja auch leicht einzusehen, dass der wandel von einem alveolaren $r$ (ein solches haben ja auch die Basler einst besessen) zu einem, sagen wir ganz allgemein rückwärtsarticulierten $r$ zunächst eintrat, wo rlickwärtige articulation der nachbarlaute bestand.

Obige hypothese kann nur bestehen, wenn zur zeit der wirkung des sandhi die auslautenden $r$ noch vorne articuliert wurden.

Irgend eine ähnliche erklärung scheinen mir auch arsch, hirsche (Seiler s. 244) zu fordern. Bei herschen könnte man allerdings auch noch beeinflussung durch das nomen herschaft annelimen.

\section{Anhang.}

$\S 62$. Der vollständigkeit wegen mögen auch die $s$ berlicksichtigung finden, die nicht in den bebandelten consonantenverbindungen vorkommen und auch nicht auf $s c$ zurtickgehen. Die wörter, welche derartige $\check{s}$ besitzen, sind entweder dem heutigen hochdeutsch oder der mundart eigentumlich, oder sie treten sporadisch in fruherer zeit auf; bei diesen letzteren ist das sch gewiss oft nur als orthographische variante fur $s$ zu betrachten.

$\S 63$. I. $\zeta$ wird ausserdem gesprochen:

1. In der 'schriftsprache' in groschen m., mhd. gros, grosse aus mlat. grossus; grosch neben gross (Tucher s. 102 bezw. 101; im 15. jh. uberhaupt schwanken zwischen diesen zwei formen, vgl. Lexer 1, 1093. Schmeller WB. 1, 1014.1)

2. In den mundarten: abgesehen von allgemeinem übergang $\operatorname{des} s$ in $s$ in Wallis (Rapp, D. Ma. 3,67), Davos, Vorarlberg

1) Hieher auch schimmel ('bureaukratischer schimmel'), das wohl auf das simile der amtssprache zurückgeht. 
(Birlinger, Al. spr. 132)1) in: schunst sonst ('und sol schunst nicht daraus prechen' Mon. Boica 10,191 ad 1472) Schmeller, Wb. 2, 433. Schm., Gr. §650 Ostlech; - felš fels Salzungen (wegen felsspalte?) - amšl amsel (Schm., Gr. § 655 Ostlech. Nassl, Tepler ma. 14. Noe, Iglauer ma., D. Ma. 5,515) wohl durch suffixübertragung von drošl drossel bewirkt; vgl. die ubertragung des suffixes -uldr -aldr vom germ. apludra-, ags. apuldre, an. apuldr 'apfelbaum' auf ags. mapuldre, ahd. hiufaltar, Kluge, Nominale stammbild. § 94. (hierzu Winteler, Kerenzer ma. s. $411 \mathrm{ff}$. Beitr. $14,455 \mathrm{ff} . \quad k+-a z z=$ got. atj $>z k>t s c h) .-\check{s}$ bei 'sollen' (oberpfälzisch. Sette communi, Schm. 3, 349. Cimbr. wb. 166, vgl. Weinhold, Mhd. gr. ${ }^{2} \S 411$. AG. § 379. BG. § 327; zur erklärung s. v. Fierlinger KZ. 27,190 ff. Johansson Beitr. 14, 290 ff.)

$\S 64$. II. sch findet sich geschrieben und vielleicht auch gesprochen :

a) bewirkt durch ein sch, das im worte vorkam: $\alpha$ ) wenn es vorausgieng: schusschiln $=$ schüsseln ${ }^{2}$ ) Breslauer urk. no. 289; geschossche $=$ geschosse Cod. dipl. Sil. 4, 258. 259; schosschen = schossen Stolle 67 ; schischel = schüssel im fränk. kochbuch hs. 18909 d. germ. mus. f. 6. 7. $10^{2}$ (Birlinger, Al. spr. 133 anm.; Pietsch bei Ruckert 144); $-\beta$ ) wenn es nachfolgte: Schalmenschiler Voc. lat. teut. hs. 57 (Birlinger 132); sleschischem silesiaco Script. rer. Sil. 6, 71 (Rückert 144); reuschisch = reussisch Osw. v. Wolkenstein 1, 2. 6; das ruschi/s land Richental 50; geschelschaft Ringk $7{ }^{\circ}, 7$. Stolle $2 .^{3}$ )

b) in sonstigen fällen: Elschi n. pr. Basler urk. no. 455 anno 1384 ; möschin messing, Glarver urk. no. 160 anno 1419; mösching Hugo v. Montfort 31, 98 (Mhd. wb. 2, 1. 159 ${ }^{\mathrm{b}}$, sch

1) Dazu vergl. auch noch Bavaria 3, 210: 'In Wülfertshausen und Mellrichstadt werden $s$ und sch wie das englische sh gesprochen und es ist ein unverkennbar leichter zischlaut. Básh, hat shi mein shuh net geshän 'base! hat sie meine schuh nicht gesehn' (regierungsbezirk Unterfranken und Aschaffenburg).

2) Hier sei auf den analogen vorgang des indischen und litauischen hingewiesen (Brugmann $\S 557,4$. $\$ 587,2$ ), z. b. ind. çváçura- aus *svaçura-, lit. szészura-s aus *seszura-s 'schwiegervater'.

8) Hieher gehören auch aus heutigen ma. paschasche passageW einhold, D. dialektf. 81; ummschuscht umsonst Schmell. Wb. 2, 333, dem sich das oben erwähnte ostlechische šunšt anschliessen dürfte. 
wegen sch in 'mischen'?); losch los: ledig und losch Zs. f. gesch. d. Oberrh. 9, 186 a. 1342 ; schuochent quaerimus, Els. pred., Alem. 1, 281; geschendet gesendet Alexander B. v. 825; mit schulchim vndirscheyd Meissner urkb. no. 34 anno 1329; Mathiasch, waisch Sendersche chron. 1530-40 (Birlinger, Augsburger ma. s. 19); grosschem Stolle 64 .

Hierzu kommen noch: heischen fur heissen $\left.{ }^{1}\right):$. . da wonden ... zwene gebroeder ... der eyne heysche Hoderich Karl Meynet 30 ; ein riter ... hisch zu sammene sine man ... vnnd hisch sy by liebe vnnd by fute, das sy alle ore habe sulden trage... Stolle 15; der bischof ... habe dem herzogen vorhei/schen vnd gelobet ib. 66; die selbe porte heischet Policastro Katzenellenbogen 368; eptischin für äbtissin ${ }^{2}$ ) (ungeınein häufig, z. b. Basler urk. 1335 no. 298. Glarner urk. 1303. 1330. 1340 etc. St. Gallen 1317. 1320 etc. Oberrhein 10,471. 479 etc. Wackernagel, Altd. pr. 122. Boner, Benecke 48, 20; eptisschin Stolle 138. Vgl. ausserdem Weinhold, Mhd. gr. ${ }^{2} \S 110$ A $\left.\S 193 \mathrm{~B} \S 154\right){ }^{3}$ )

$\S 65$. Andrerseits findet sich für sch aus sc öfters $s$ geschrieben.

1. Vor $r$ : Dietrich der Sreiber Urk. v. Oberōst. 5, 164 a. 1316; srift Wackern., Altd. pr. 13,23. Vgl. auch Rlickert 142. Weinhold a. a. 0 .

2. Vor -heil: wenn kusheit, mensheit Ruckert 142, mennisheit Wack. pr. 2, 7, jüdiesheit Register 48. 56. 57. 72. 74. 78 nicht als kuscheit etc. aufzufassen sind; sh fur sch oft vorkommende ortbographische variante.

3. vleis fur vleisch (wurde [noch heute flass und friss frisch im nassauischen, Kehrein no. 171] gewiss auch in einigen gegenden so gesprochen, $\nabla$ gl. auch ndl. vleezig 'fleischig' ohne $k$-ableitung) Ruckert a. a. 0., Augsb. stadtb. 198, 26, fleifs Stolle 95. In der zusammensetzung in: fleishackel Augsb. stb. 31. 32. 34. 198. 199 etc.

1) An der Blies wird héscho sogar für hai/sen gebraucht, Schmeller Wb. 1, 1184 .

2) abschtischn bei Gryphius, elsïss. abtischin, ndd. ebbedische Weinhold, D. dialektf. 81.

s) $\dot{s}$ bezw. $\check{z}$ für $s$ zeigt sich im Wiener jargon in wižərln, vgl. wiseln, wis wis machen, wissern Schmell. BW. 2, 1039. Vilmar, Kurhess. idioticon 456 ; zu visel, stm. membrum visile? 
vielleicht analog wie oben in 2) aufzufassen, rgl. fleischakerzech Urk. v. Oberöst. 8, 37 anm. 1367; zur orthographischen verschmelzung zweier ch vgl. nachomen für nachchomen 1370, ib. 8, 471; Erlachlohter für Erlachchloster ib. 8, 327.

4. -es, is fur -esch, isch: hubes hl. Elis. 167; kindes ib. 1543; romes ib. 7870.

Ferner: sachare Wack., Pr. 37. 39; sulmilch Dankrotzh. 319 ; chusir Wack. pr. 75, 2; men/sin ib. 2,53; inzwissen 1, 103; harna/s Stolle 141; fis piscis hl. Elis. 1109; disse dat. v. tisch ib. 2519. 2719. 3647 im reime auf gewisse certe 1717. 2123; dissegader m. tischgenosse ib. 2727; blamensier, -sir, -ser für blâmenschir blanc-manger (vgl. hl. Georg v. Reinbot v. Durne 1913 'swie doch ein fürste dâ was gast, blâmenschire was dâ tiure'. Buch von guter speise 3. 76. 77. Es ist wohl in vielen dieser fälle (bl. Elisabeth: Wetterau) wie in den mnd. schreibungen vals, valsk, valsch, vis, vlês (?), harnas, Riges, Rigisch, Lives (Livisch) etc. (Lubben, Mnd. gr. § 35) das $s$ eine orthographische variante des sch.

WIEN, 2. september 1891.

OTTO ARON. 